\title{
1 CHOmpact: a reduced metabolic model of Chinese hamster ovary cells
} with enhanced interpretability

Ioscani Jiménez del Val1,*, Sarantos Kyriakopoulos²,ł, Simone Albrecht³, Henning Stockmann³, Pauline M Rudd ${ }^{3, \dagger}$, Karen M Polizzi ${ }^{4}$ and Cleo Kontoravdi ${ }^{4}$

${ }^{1}$ School of Chemical \& Bioprocess Engineering, University College Dublin D04 V1W8, Ireland ${ }^{2}$ MS\&T, BioMarin Manufacturing Ireland, Cork P43 R298, Ireland

${ }^{3}$ NIBRT GlycoScience Group, National Institute for Bioprocessing Research and Training, Dublin A94 X099, Ireland

${ }^{4}$ Department of Chemical Engineering, Imperial College London SW7 2AZ, United Kingdom *Author to whom correspondence should be addressed. e-mail: ioscani.jimenezdelval@ucd.ie

† Present address: Bioprocessing Technology Institute, Agency for Science, Technology and Research (A*STAR) 138668, Singapore ‡ Present address: Process Development, Lonza Group AG, Wisp 3930, Switzerland 


\section{Abstract}

Metabolic modelling has emerged as a key tool for the characterisation of biopharmaceutical cell culture processes. Metabolic models have also been instrumental in identifying genetic engineering targets and developing feeding strategies that optimise the growth and productivity of Chinese hamster ovary (CHO) cells. Despite their success, metabolic models of CHO cells still present considerable challenges. Genome scale metabolic models (GeMs) of CHO cells are very large (>6000 reactions) and are, therefore, difficult to constrain to yield physiologically consistent flux distributions. The large scale of GeMs also makes interpretation of their outputs difficult. To address these challenges, we have developed CHOmpact, a reduced metabolic network that encompasses 101 metabolites linked through 144 reactions. Our compact reaction network allows us to deploy multi-objective optimisation and ensure that the computed flux distributions are physiologically consistent. Furthermore, our CHOmpact model delivers enhanced interpretability of simulation results and has allowed us to identify the mechanisms governing shifts in the anaplerotic consumption of asparagine and glutamate as well as an important mechanism of ammonia detoxification within mitochondria. CHOmpact, thus, addresses key challenges of large-scale metabolic models and, with further development, will serve as a platform to develop dynamic metabolic models for the control and optimisation of biopharmaceutical cell culture processes.

\section{Introduction}

Production of recombinant proteins is known to compete with biomass synthesis for externally provided nutrients. This is particularly true for mammalian cell lines, such as Chinese hamster ovary (CHO) cells, which are the dominant host for industrial production of therapeutic proteins (O'Flaherty et al., 2020). Metabolic modelling has become an essential tool for understanding resource allocation and, coupled with advances in genome editing, designing rational cell engineering strategies. Publication of the $\mathrm{CHO}-\mathrm{K} 1$ genome, and the omics analyses this enabled, laid the foundation for systems-level understanding of this host. This knowledge has been reconstructed mathematically in a community-curated genome-scale metabolic model (GeM) of 
the CHO cell termed iCHO1766 (Hefzi et al., 2016). Crucially, the GeM organised knowledge of all biochemical conversions, transport and exchange reactions to create a large, interlinked network of metabolites and their associated reactions.

The inclusion of gene-protein reaction associations provided a direct link between genes and metabolic reactions. Since then, significant expansions and improvements to iCH01766 have been achieved, such as gap-filling studies that also removed dead-end reactions (Fouladiha et al., 2021), and the integration of a core protein secretory pathway, iCHO2048, enabling the computation of energetic costs and machinery demands of each secreted protein (Gutierrez et al., 2020). Interestingly, iCHO2048 was subsequently used to direct host cell protein knockout studies which resulted in increased recombinant protein productivity and a cleaner feedstock for downstream processing steps (Kol et al., 2020), highlighting the power these models hold for identifying diverse cellular engineering strategies.

The solution of GeMs, and any undetermined metabolic model, relies on constraint-based methods, such as flux balance analysis (FBA), to predict steady-state intracellular flux distributions (Orth et al., 2010). Although FBA offers the advantage of not requiring detailed knowledge of enzymatic kinetic parameters, it does not return a unique set of intracellular flux values. In addition, the larger the metabolic network considered, the more difficult it becomes to interpret such predictions (Gardner and Boyle, 2017). GeMs therefore require large datasets, preferably across different omics levels (e.g., metabolomic, transcriptomic) to increase confidence in results. This is also true for curating GeMs for specific cell lines or systems, raising the need for extensive experimentation that goes beyond typical analytical measurements conducted in an industrial setting.

Several algorithms have been developed to improve the predictive performance of GeMs, for example by constraining the amount of carbon able to flowthrough reaction fluxes, based on the maximum amount carbon uptake by the cell (ccFBA) (Lularevic et al., 2019), taking into account the selective pressure that exists within cell cultures for fast-growing cell lines with a low enzyme 
usage (Lewis et al., 2010), or introducing enzyme capacity constraints (Yeo et al., 2020). Despite these advances, both the accuracy and interpretability of intracellular flux predictions remain challenging. An additional limitation is the computational difficulty in creating dynamic versions of GeMs that would reflect the nature of cell culture processes, although recent efforts coupling a CHO GeM with statistical models have yielded promising results in predicting the time evolution of extracellular amino acid concentrations (Martínez et al., 2015).

In this work, we introduce a reduced-scale metabolic model, CHOmpact, where the reaction network is based on the work by Carinhas et al. (2013) and has been augmented with a detailed description the aspartate-malate (Asp-Mal) shuttle, the urea cycle, de novo serine synthesis from glycolytic intermediates, and nucleotide sugar donor biosynthesis. The resulting network comprises 101 metabolites and 144 reactions, which due to its compact nature, significantly enhances the interpretability of simulation results. The reduced scale of the network and associated FBA problem also allow for more complex, non-linear formulations of the objective function to be incorporated compared to biomass maximisation that is often employed in FBA of GeMs. The multi-objective optimisation framework used to solve CHOmpact allows us to solve across all phases of cell culture and provides insight into the dynamics of cellular metabolism. We envisage that the advantages presented by CHOmpact will enable the development of dynamic flux balance models that can serve as digital twins for the control and optimisation of biopharmaceutical cell culture processes.

\section{Materials \& Methods}

\subsection{Experimental}

\subsubsection{Cell culture}

The GS46 GS-CHO cell line producing a humanized anti-Tumour-Associated Glycoprotein (TAG72) IgG4א mAb (cB72.3), a kind gift by Lonza Biologics (Slough, UK), was cultured with three different amino acid feeds: Feed C, Feed U and Feed U40 (Kyriakopoulos and Kontoravdi, 2014). Briefly, triplicate cultures for each feeding regime were performed in orbitally shaken (140 rpm) 
$250 \mathrm{~mL}$ vented conical flasks (Corning, Amsterdam, Netherlands) with a 50mL working volume. The cultures were performed in a humidified incubator with $\mathrm{CO}_{2}$ controlled at $8 \%$ and temperature set at $36.5^{\circ} \mathrm{C}$. The basal culture medium for all cultures was $\mathrm{CD}$ CHO (Life Technologies, Paisley, UK) supplemented with $25 \mu \mathrm{M}$ methionine sulfoximine (Sigma-Aldrich, Dorset, UK). All feeding regimes consisted in adding 10\% v/v every 48 hours of culture starting on day 2. The Feed $\mathrm{C}$ regime used commercial CD EfficientFeed ${ }^{\mathrm{TM}} \mathrm{C}$ AGT $^{\mathrm{TM}}$ (Invitrogen, UK), whereas the $\mathrm{U}$ and $\mathrm{U} 40$ feeds aimed to provide growth-limiting nutrients (glucose and amino acids) beyond the amounts available in Feed C. The glucose and amino acid concentrations present in the different feeds is detailed in previous work by Kyriakopoulos and Kontoravdi (2014).

\subsubsection{Analytical methods}

Viable and dead cell density was determined using the trypan blue dye exclusion method and light microscopy. mAb titre was determined using the BLItz ${ }^{\circledR}$ system (Pall ForteBio, Portsmouth, UK). Time profiles for glucose, lactate, and ammonia were generated using the Bioprofile 400 analyser (NOVA Biomedical, Waltham, MA). Residual amino acid profiles were generated using the PicoTag method (Waters, Hertfordshire, UK) on an Alliance HPLC instrument (Waters, Hertfordshire, UK). Extracellular pyruvate concentrations were determined with an enzyme assay kit (Abcam, Cambridge, UK). mAb Fc glycoprofiling was performed with an automated sample preparation workflow (Stockmann et al., 2013). Briefly, the mAb samples were affinitypurified from the cell culture supernatant with a 96-well Protein G IgG purification plate (Thermo Fisher Scientific, Dublin, Ireland). Glycans were released from the mAb through PNGase (Prozyme, Hayward, California) digestion and labelled with 2-amino benzamide (Ludger, Oxford, UK). Labelled glycans were separated using ultra-performance hydrophilic interaction chromatography (UPLC-HILIC) and quantified through fluorescence detection (Stockmann et al., 2013). Glycans were initially assigned by comparing their Glucose Unit retention times with those available in the NIBRT GlycoBase 3.2 structural N-glycan library (Campbell et al., 2008). Glycan 
assignment was confirmed through weak anion exchange chromatography and quadrupole timeof-flight mass spectrometry on exoglycosidase-digested samples (Albrecht et al., 2014).

\subsubsection{Dry cell weight measurement}

126 Cells were cultured under the same conditions as described for Feed C, above. Duplicate cultures determined using the trypan blue dye exclusion method. Immediately after cell counting, $40 \mathrm{~mL}$ of the cultures was harvested and centrifuged at $1000 \mathrm{~g}$ for one minute in pre-weighed $50 \mathrm{~mL}$ falcon tubes. The supernatant was then discarded, and the cell pellets were washed once with 40mL 0.9\% w/v NaCl (Sigma-Aldrich, Dorset, UK) solution and centrifuged at $1000 \mathrm{~g}$ for one minute. The wash was discarded, and the cell pellet was left to dry in a non-humidified incubator at $37^{\circ} \mathrm{C}$ until no changes in weight were observed. The tubes were weighed within $1 \mathrm{mg}$ accuracy (ACCULAB, Sartorius, Surrey, UK).

\subsubsection{Data processing and analysis}

The cell-specific rates for nutrient consumption and metabolite/product secretion, $q_{i}\left(t_{n}\right)$, were calculated by performing linear regressions to obtain the slopes in Eq. 1 (Sauer et al., 2000), where $N_{i, c o n s}\left(t_{n}\right)$ is the consumed/produced amount of component $i$ (in units of $n m o l_{i}$ or $m g_{m A b}$ ) up to time $t_{n}$ and $I V C\left(t_{n}\right)$ is the integral of viable cells up to time $t_{n}$ in units of $10^{6} \mathrm{cells} \mathrm{h}$.

$$
\begin{aligned}
& N_{i, \text { cons }}\left(t_{n}\right)=q_{i}\left(t_{n}\right) \operatorname{IVC}\left(t_{n}\right) \\
& N_{i, \text { cons }}\left(t_{n}\right)=[C]_{i, \text { res }}\left(t_{n}\right) \operatorname{Vol}\left(t_{n}\right)-\sum_{j=1}^{n}[C]_{i, \text { feed }} \operatorname{Vol}_{f e d}\left(t_{j}\right) \\
& \operatorname{IVC}\left(t_{n}\right)=\sum_{j=1}^{n} \frac{\left[X_{v}\right]\left(t_{j}\right) \operatorname{Vol}\left(t_{j}\right)+\left[X_{v}\right]\left(t_{j-1}\right) \operatorname{Vol}\left(t_{j-1}\right)}{2}\left(t_{j}-t_{j-1}\right)
\end{aligned}
$$

$141 N_{i, \text { cons }}\left(t_{n}\right)$ and $\operatorname{IVC}\left(t_{n}\right)$ were computed using Eq. 2 and Eq. 3, respectively. In Eq. 2, $[C]_{i, r e s}\left(t_{n}\right)$ is 
$i$ in the feed and $\operatorname{Vol}_{f e d}\left(t_{j}\right)$ is the feed volume added at time $j$. In Eq. 3, $\left[X_{v}\right]\left(t_{j}\right)$ is cell density at

144 time $t_{j}$ and $\operatorname{Vol}\left(t_{j}\right)$ is liquid volume in the culture flask at time $t_{j}$.

145 The linear regressions for the determination of cell specific uptake rates were computed using 146 the LINEST function in Microsoft Excel. Confidence intervals for the obtained $q_{i}\left(t_{n}\right)$ values were 147 computed with the least square residuals and a t-value for $p=0.05$. Data analysis identified six 148 distinct intervals with constant uptake/secretion rates: early exponential, mid-exponential, late 149 exponential, early stationary, and stationary. Raw cell culture data is presented in Supplementary Figure 1, and the processed data, along with the identified intervals are presented in Supplementary Figure 2.

The amount of mAb glycoform secreted across different intervals was calculated using Eq. 4, where $f_{k}$ represents the fraction of mAb glycoform $k$ secreted during the time interval $\left(t_{n}-t_{n-1}\right),[m A b]_{i}\left(t_{n}\right)$ is the concentration of mAb glycoform $k$ present at time $t_{n}$ and $[m A b]\left(t_{n}\right)$ is the total $\mathrm{mAb}$ titre at time $t_{n}$ (del Val et al., 2016a; Fan et al., 2015).

$$
f_{k}=\frac{[m A b]_{k}\left(t_{n}\right)-[m A b]_{k}\left(t_{n-1}\right)}{[m A b]\left(t_{n}\right)-[m A b]\left(t_{n-1}\right)}
$$

\subsection{Flux balance model development}

157 Our flux balance model (Figure 1) is based on previous work for GS-CHO cells (Carinhas et al., 158 2013), which has been expanded to include the aspartate-malate (Asp-Mal) shuttle (Mulukutla et 159 al., 2012; Nolan and Lee, 2011), the urea cycle (Zamorano et al., 2010), de novo serine synthesis 160 from glycolytic intermediates, and ATP synthesis via oxidative phosphorylation. The pathway for 161 nucleotide sugar donor biosynthesis (Kremkow and Lee, 2018) has also been included.

162 Manual curation of our FBA model was performed using the KEGG database (Kanehisa et al., 2017; Kanehisa et al., 2019) and the reference CHO-K1 and Cricetulus griseus genome annotations (Kremkow et al., 2015; Lewis et al., 2013; Rupp et al., 2018). The resulting model comprises 101 metabolites linked through 299 reactions (Supplementary Table 1). Supplementary Table 1 also 


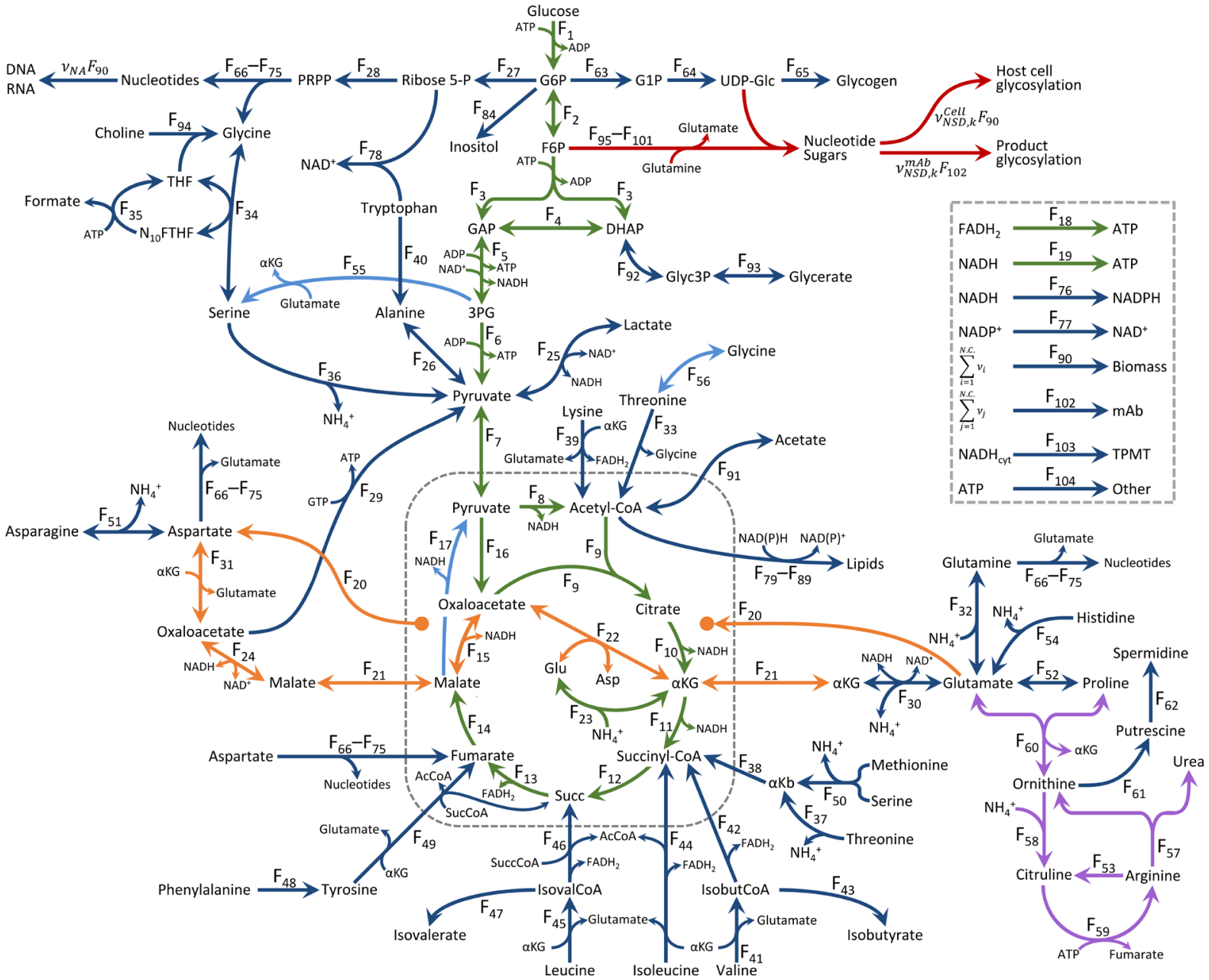

Figure 1. FBA reaction network

The present FBA model considers 101 species linked through 144 fluxes. Different colours indicate

Sequential reactions throughout the metabolic pathways were combined into single reaction fluxes to reduce degrees of freedom within the model (Nolan and Lee, 2011). Overall, the resulting metabolic model considers material balances for 101 species, one additional equation that defines the consumption of ATP towards active amino acid transport and 144 fluxes (Figure 1 
and Supplementary File, Section 2), yielding 42 degrees of freedom. The full stoichiometric matrix underlying our model is presented in Supplementary Table 2.

\subsubsection{Stoichiometric equations for biomass and product}

182 Calculations for the biomass stoichiometric coefficients are presented in Supplementary Table 3. Based on experimental measurements, the biomass stoichiometric equation considers a dry cell weight of $219 \mathrm{pg} /$ cell for exponentially growing cells and $311 \mathrm{pg} /$ cell for cells in stationary phase. The mass composition of GS-CHO cells is based on Sheikh et al. (2005) and Hefzi et al. (2016) and assumes $74.2 \%$ protein, $11.1 \%$ lipids, $5.0 \%$ RNA, $1.4 \%$ DNA, $0.4 \%$ glycogen, $0.2 \%$ N-glycans, $0.3 \%$ O-glycans, $2.9 \%$ other intracellular components (e.g. MTHF, NAD(P)H, AcCoA) and 4.5\% nonbalanced components (ash).

The individual amino acid content of protein was computed from $\mathrm{CHO}$ cell proteomic data

190 (Baycin-Hizal et al., 2012), as reported previously (del Val et al., 2016b). The glycan content of 191 biomass, which has been included by using the biomass NSD stoichiometric coefficients for 192 cellular protein N- and O-linked glycosylation as well as glycolipid glycosylation (del Val et al., 193 2016b).

194 The stoichiometric equation for the cB72.3 product, a humanised IgG4 $\kappa$ mAb, was computed 195 based on the amino acid sequences for the human IgG4 Fc (Heilig et al., 2003), the constant 196 fragment of a human kappa light chain (Brady et al., 1991; Xiang et al., 1999), as well as the 197 variable heavy and light chain fragments for the cB72.3 mAb (Xiang et al., 1999). The sequences 198 and calculations for the mAb amino acid stoichiometric coefficients are presented in 199 Supplementary Table 4. mAb glycoprofiling at three culture timepoints (192h, 240h, and 288h) allowed us to calculate stoichiometric coefficients for NSD consumption towards mAb glycosylation across three culture intervals: 0 to 192 hours, 192 to 240 hours, and 240 to 288 hours. These calculations were made with Eq. 4, and the obtained stoichiometric values are presented in Supplementary Table 5. 


\subsubsection{FBA solution: multi-objective optimisation}

As with most FBA models, no intracellular accumulation of species has been assumed in the material balances generated from our stoichiometric matrix, leading to a problem of the form:

$$
S \times F=0
$$

Eq. 5

Where $S$ is the stoichiometric matrix defined in Supplementary Table 2 and $F$ is the vector of unknown fluxes. Because the model contains more unknown fluxes (144) than equations (102), it must be solved using constraint-based optimisation strategies that have been outlined elsewhere (Banga, 2008).

Two constraint-based optimisation strategies have been used to solve our FBA. The first is typical in that it maximises the rate of biomass synthesis while maintaining the transport flux for all nutrients, metabolites, and product set to their experimentally determined values. Reaction reversibility constraints, based on enzyme data available in the KEGG (Kanehisa et al., 2017; Kanehisa et al., 2019) and BRENDA (Jeske et al., 2019) databases, were included and are indicated in Supplementary Tables 1 and 2.

The multi-objective optimisation strategy proposed herein simultaneously maximises the fluxes where ATP is synthesised while minimising the sum of squared intracellular fluxes. This objective function represents maximum energetic efficiency by the cells (Schuetz et al., 2007) and was used to ensure consistent directionality of central carbon metabolism fluxes. Alongside maximising the energetic efficiency of the cells, the squared difference between measured and computed fluxes was minimised to ensure consistency between our flux model results and our experimental measurements. In order to avoid flux $F_{15}$ being bypassed by $F_{17}$, the $F_{17} / F_{14}$ ratio was constrained to values within the $[0,1]$ interval and minimised. Finally, the sum of non-measured by-product secretion fluxes was also minimised as part of this multi-objective optimisation strategy. As with the traditional optimisation strategy, flux reversibility constraints were included within our multi-objective optimisation strategy. 
An additional constraint was included for the maximum allowable fluxes along the aspartatemalate (Asp-Mal) shuttle. Flux through the Asp-Mal shuttle was constrained by limiting the maximum amount of glutamate transported into the mitochondrial lumen $\left(F_{20}\right)$ to the flux of 231 glutamate internalised by the cells or produced through reactions independent of the Asp-Mal shuttle. $F_{20}$ was constrained because this antiport transport flux is the rate-limiting step of the Asp-Mal shuttle (LaNoue et al., 1974; LaNoue and Tischler, 1974).

Importantly, a constraint for Asp-Mal fluxes is required because the sum of all reactions in the shuttle result, exclusively, in net transport of cytosolic NADH into the mitochondrial lumen (see Supplementary File). Thus, the fluxes through the reactions underlying the Asp-Mal shuttle can take any value as long as the balances for cytosolic and mitochondrial NADH are met (i.e., all other fluxes cancel each other out). This is likely to be the cause of what may be considered high (and possibly inconsistent) Asp-Mal shuttle fluxes with values that are comparable with glucose uptake fluxes (Mulukutla et al., 2012; Nolan and Lee, 2011). Avoiding these potential inconsistencies led us to include this Asp-Mal constraint within our FBA solution strategy.

Our multi-objective optimisation strategy also constrains $F_{16}$ to be $1 \%$ of the glucose uptake flux growth (Ahn and Antoniewicz, 2013). Both optimisation strategies are outlined in Table 1, where

248 (a reaction is irreversible when $L B_{i}=0$ ), $C_{A s p / M a l}$ is the Asp-Mal shuttle constraint, $\varepsilon$ represents

249 a small threshold value, $F_{m}^{\text {Comp. }}$ and $F_{m}^{\text {Meas. }}$ are the computed and measured transport fluxes, 250 respectively, $F_{g}$ are the fluxes of reactions where Glu is produced or consumed (excluding Asp251 Mal shuttle reactions), and $v_{g}$ is the stoichiometric coefficient for these Glu synthesis reactions. 


\begin{tabular}{|c|c|}
\hline BM maximisation & Multi-objective optimisation \\
\hline $\begin{array}{c}\operatorname{MAX}\left(F_{142}\right) \\
\text { Subject to: } \\
{\left[\begin{array}{c}L B_{i} \leq F_{i} \leq U B_{i} \\
{\left[100\left(\frac{F_{143}}{q_{p}}-1\right)\right]^{2} \leq \varepsilon_{p}}\end{array}\right.}\end{array}$ & 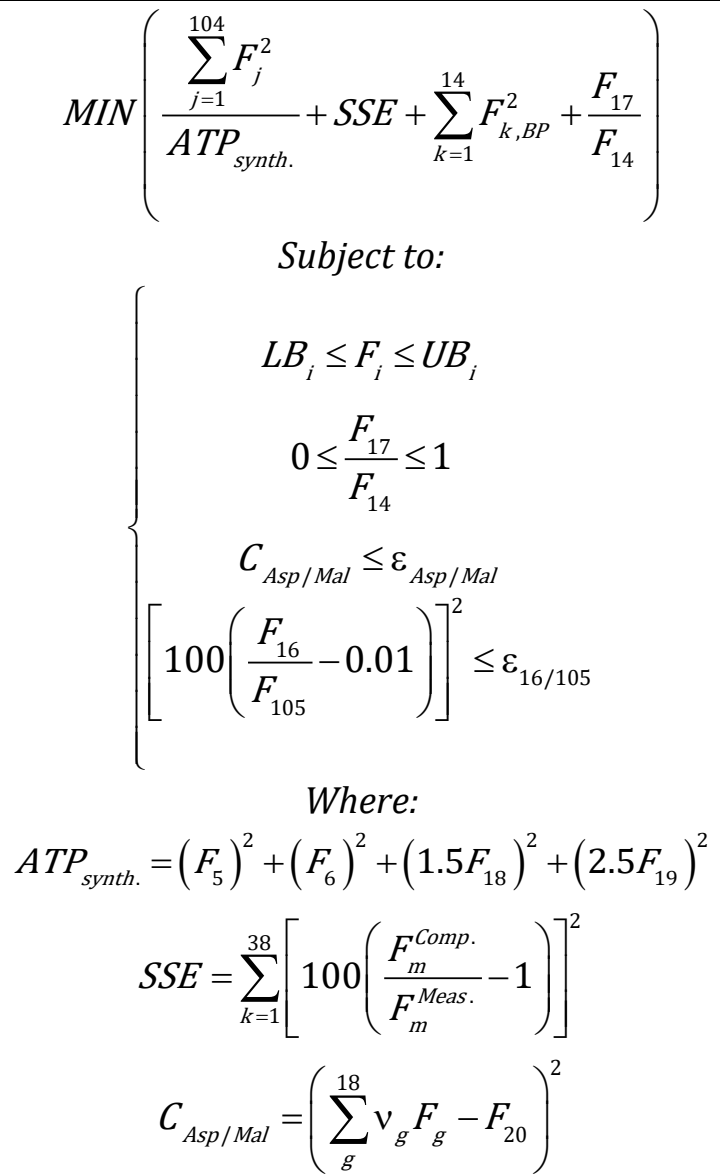 \\
\hline
\end{tabular}

The biomass maximisation strategy was used to compare performance of our reduced FBA model with the iCH01766 GeM (Hefzi et al., 2016) while the energetic efficiency maximisation strategy was used for all other simulations presented herein. All optimisations were performed using the nonlinear programming sequential quadratic programming (NLPSQP) solver built into gPROMS ModelBuilder v6.0.2 (Process Systems Enterprise) on a standard desktop workstation (AMD Ryzen 2700x@ 4GHz and 16GB RAM).

\section{Results and discussion}

\subsection{The reduced reaction network performs comparably with the iCHO1766 GeM}

The maximum specific growth rates $\left(\mu_{g, \max }\right)$ of multiple CHO cell lines cultured under different conditions were calculated through the biomass synthesis rate maximisation strategy. The 
required inputs for this solution strategy, namely the nutrient, metabolite, and product transport

In order to assess whether our assumed biomass composition and reduced reaction network performs comparably with a large-scale model for $\mathrm{CHO}$ cell metabolism, Figure 2 compares our predicted $\mu_{\text {g,max }}$ values with those obtained using the iCHO1766 GeM (Hefzi et al., 2016) and the corresponding experimental data.

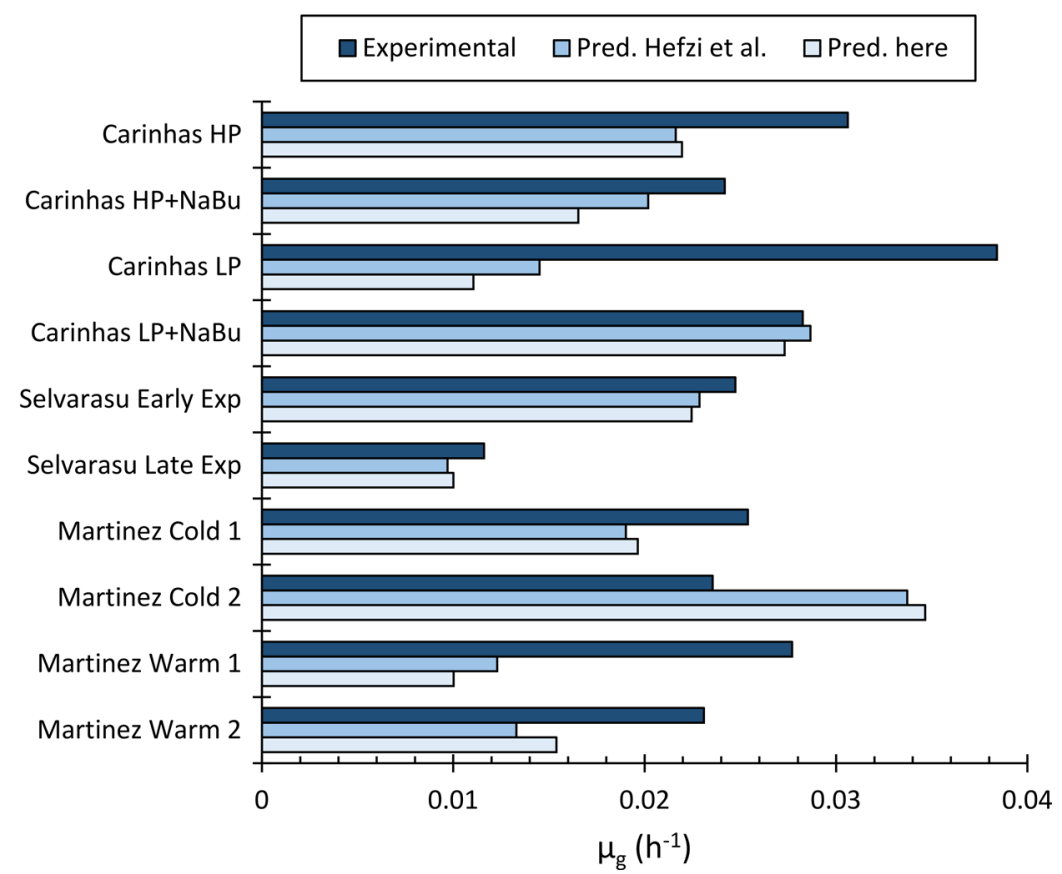

Figure 2. Comparison of experimentally determined and predicted maximum specific growth rates experimentally determined $\mu_{g, m a x}$ values reported by Carinhas et al. (2013), Martínez et al. (2015), and Selvarasu et al. (2012). The medium blue bars present $\mu_{g, \max }$ predictions reported by Hefzi et al. (2016) and the light blue bars show the $\mu_{g, \max }$ values predicted with CHOmpact. for the Low Producing $\mathrm{CHO}$ cell line cultured with sodium butyrate $(\mathrm{LP}+\mathrm{NaBu})$ from Carinhas et al. (2013) as well as the early and late exponential growth phases reported by Selvarasu et al. 

as well as CHO cells cultured under normal and mild hypothermic conditions (Warm 1, Warm 2, and Cold 1, respectively) (Martínez et al., 2015). Our model overpredicts the biomass synthesis rate of the second hypothermic dataset (Cold 2) reported by Martínez et al. (2015).

$\mu_{g, \max }$ optimisations were performed using the biomass composition assumed by Hefzi et al. caused by our assumed biomass composition. These optimisations yield overall average deviations from the experimental data that are indistinguishable from those obtained with our assumed biomass composition (data not shown). These results are expected, especially when considering that the minor differences between our assumed biomass composition and the one used in the GEM involve prototrophic amino acids (Ala, Gln, Gly) (Supplementary Figure 4).

Although the deviations are considerable in some cases, our predictions are similar to those reported for the CHO GeM (Hefzi et al., 2016), and are better for Carinhas HP, Selvarasu Late Exp, Martinez Cold 1, and Martinez Warm 2. Overall, the GeM has an average percent deviation across the ten experimental $\mu_{g, \max }$ values of $30 \%$, whereas our model has an average deviation of $32.5 \%$. When considering that our model includes only 144 reactions when compared to 6,663 in the GeM, a $2.5 \%$ reduction in predictive capability is acceptable, especially considering gains in model output consistency and interpretability (to be discussed in subsequent sections) as well as reductions in computational expense, when using non-linear, multi-objective optimisation strategies for solution.

\subsubsection{CHOmpact identifies the source of $\mu_{g}$ prediction inaccuracies}

302 An advantage of our proposed multi-objective optimisation strategy is that it enables the identification of nutrient uptake rates that lead to shortfalls in calculated specific growth rates when compared with the experimental values. This is achieved by constraining the maximum allowable value for the percent error of biomass growth rate and specific productivity to low values $\left(5 \times 10^{-6}\right)$ while fixing the uptake/secretion flux values of Glc, Lac, $\mathrm{NH}_{4}{ }^{+}$, Pyr, Ala, Asn, Asp, 
307 Glu, Gly to the experimentally determined flux values. The upper bounds for the uptake fluxes of

308 the remaining (mainly auxotrophic) amino acids are relaxed and obtained via constrained

309 optimisation where the SSE is minimised. This strategy ensures that the optimal solution matches

310 the experimental growth rate and specific productivity while finding the combination of amino

311 acid uptake fluxes that minimises deviations from the experimental uptake rates.

312 The above strategy yields the results presented in Table 2, where the percent increases in specific

313 uptake rates required for matching the experimentally determined $\mu_{g}$ and $q_{p}$ are shown

314 (positive/red values denote percent increase in uptake fluxes required to match $\mu_{g}$ and $q_{p}$ ). Table

3152 shows that for Car LP (NaBu), Selv (Early), Selv (Late), and Mart (Cold2), small or no increases

316 in amino acid uptake rates are required to match $\mu_{g}$ and $q_{p}$. This is expected, considering the

317 results of Figure 2, where the predicted values for $\mu_{g}$ are matched or exceeded for these datasets.

318 Table 2. Percent increases in amino acid uptake rates required to match $\mu_{\mathrm{g}}$ and $\boldsymbol{q}_{p}$. The intensity of

319 the colours (from green $=\mathbf{0} \%$ to red) corresponds to magnitudes across all datasets.

\begin{tabular}{|c|c|c|c|c|c|c|c|c|c|c|}
\hline & Car HP & $\begin{array}{l}\text { Car HP } \\
\text { (NaBu) }\end{array}$ & Car LP & $\begin{array}{l}\text { Car LP } \\
\text { (NaBu) }\end{array}$ & $\begin{array}{c}\text { Selv } \\
\text { (Early) }\end{array}$ & $\begin{array}{c}\text { Selv } \\
\text { (Late) }\end{array}$ & $\begin{array}{c}\text { Mart } \\
\text { (Cold1) }\end{array}$ & $\begin{array}{c}\text { Mart } \\
\text { (Cold2) }\end{array}$ & $\begin{array}{c}\text { Mart } \\
\text { (Warm1) }\end{array}$ & $\begin{array}{c}\text { Mart } \\
\text { (Warm2) }\end{array}$ \\
\hline$\mu_{g}$ & $0.0 \%$ & $0.0 \%$ & $0.0 \%$ & $0.0 \%$ & $0.0 \%$ & $0.0 \%$ & $0.0 \%$ & $0.0 \%$ & $0.0 \%$ & $0.0 \%$ \\
\hline$q_{p}$ & $0.0 \%$ & $0.0 \%$ & $0.0 \%$ & $0.0 \%$ & $0.0 \%$ & $0.0 \%$ & $0.0 \%$ & $0.0 \%$ & $0.0 \%$ & $0.0 \%$ \\
\hline$q_{\text {GIc }}$ & $0.0 \%$ & $0.0 \%$ & $0.0 \%$ & $0.0 \%$ & $0.0 \%$ & $0.0 \%$ & $0.0 \%$ & $0.0 \%$ & $0.0 \%$ & $0.0 \%$ \\
\hline$q_{L a c}$ & $0.0 \%$ & $0.0 \%$ & $0.0 \%$ & $0.0 \%$ & $0.0 \%$ & $0.0 \%$ & $0.0 \%$ & $0.0 \%$ & $0.0 \%$ & $0.0 \%$ \\
\hline$q_{N H 4}$ & $0.0 \%$ & $0.0 \%$ & $0.0 \%$ & $0.0 \%$ & $0.0 \%$ & $0.0 \%$ & $0.0 \%$ & $0.0 \%$ & $0.0 \%$ & $0.0 \%$ \\
\hline$q_{P y r}$ & $0.0 \%$ & $0.0 \%$ & $0.0 \%$ & $0.0 \%$ & $0.0 \%$ & $0.0 \%$ & $0.0 \%$ & $0.0 \%$ & $0.0 \%$ & $0.0 \%$ \\
\hline$q_{A l a}$ & $0.0 \%$ & $0.0 \%$ & $0.0 \%$ & $0.0 \%$ & $0.0 \%$ & $0.0 \%$ & $0.0 \%$ & $0.0 \%$ & $0.0 \%$ & $0.0 \%$ \\
\hline$q_{\text {Asn }}$ & $0.0 \%$ & $0.0 \%$ & $0.0 \%$ & $0.0 \%$ & $0.0 \%$ & $0.0 \%$ & $0.0 \%$ & $0.0 \%$ & $0.0 \%$ & $0.0 \%$ \\
\hline$q_{A s p}$ & $0.0 \%$ & $0.0 \%$ & $0.0 \%$ & $0.0 \%$ & $0.0 \%$ & $0.0 \%$ & $0.0 \%$ & $0.0 \%$ & $0.0 \%$ & $0.0 \%$ \\
\hline$q_{G l n}$ & $0.0 \%$ & $0.0 \%$ & $0.0 \%$ & $0.0 \%$ & $0.0 \%$ & $0.0 \%$ & $0.0 \%$ & $0.0 \%$ & $48.7 \%$ & $35.0 \%$ \\
\hline$q_{G l u}$ & $0.0 \%$ & $0.0 \%$ & $0.0 \%$ & $0.0 \%$ & $0.0 \%$ & $0.0 \%$ & $0.0 \%$ & $0.0 \%$ & $0.0 \%$ & $0.0 \%$ \\
\hline$q_{\text {Gly }}$ & $0.0 \%$ & $0.0 \%$ & $0.0 \%$ & $0.0 \%$ & $0.0 \%$ & $0.0 \%$ & $0.0 \%$ & $0.0 \%$ & $0.0 \%$ & $0.0 \%$ \\
\hline$q_{A r g}$ & $0.2 \%$ & $0.0 \%$ & $8.4 \%$ & $-0.1 \%$ & $-0.3 \%$ & $0.0 \%$ & $0.8 \%$ & $-0.4 \%$ & $17.4 \%$ & $18.6 \%$ \\
\hline$q_{\text {His }}$ & $0.5 \%$ & $25.0 \%$ & $33.9 \%$ & $0.0 \%$ & $0.0 \%$ & $2.6 \%$ & $1.8 \%$ & $0.0 \%$ & $22.7 \%$ & $6.8 \%$ \\
\hline$q_{I l e}$ & $0.9 \%$ & $0.0 \%$ & $20.2 \%$ & $0.0 \%$ & $0.0 \%$ & $0.0 \%$ & $2.5 \%$ & $0.0 \%$ & $9.5 \%$ & $13.2 \%$ \\
\hline$q_{L e u}$ & $13.0 \%$ & $1.4 \%$ & $29.8 \%$ & $0.0 \%$ & $0.4 \%$ & $9.0 \%$ & $5.7 \%$ & $0.1 \%$ & $18.6 \%$ & $16.2 \%$ \\
\hline$q_{\text {Lys }}$ & $24.7 \%$ & $0.1 \%$ & $38.8 \%$ & $0.0 \%$ & $0.0 \%$ & $4.0 \%$ & $22.0 \%$ & $0.0 \%$ & $41.0 \%$ & $18.7 \%$ \\
\hline$q_{M e t}$ & $9.4 \%$ & $0.0 \%$ & $23.4 \%$ & $0.0 \%$ & $0.0 \%$ & $0.0 \%$ & $0.0 \%$ & $0.0 \%$ & $13.8 \%$ & $0.1 \%$ \\
\hline qPhe & $25.4 \%$ & $0.0 \%$ & $47.4 \%$ & $3.3 \%$ & $8.4 \%$ & $10.1 \%$ & $16.4 \%$ & $0.1 \%$ & $33.2 \%$ & $8.1 \%$ \\
\hline$q_{\text {Pro }}$ & $0.9 \%$ & $0.0 \%$ & $21.9 \%$ & $0.0 \%$ & $0.0 \%$ & $0.0 \%$ & $2.0 \%$ & $0.0 \%$ & $2.4 \%$ & $7.3 \%$ \\
\hline$q_{\text {Ser }}$ & $3.3 \%$ & $0.1 \%$ & $41.1 \%$ & $0.1 \%$ & $0.0 \%$ & $0.0 \%$ & $9.1 \%$ & $0.2 \%$ & $25.0 \%$ & $33.4 \%$ \\
\hline$q_{T h r}$ & $3.8 \%$ & $0.0 \%$ & $15.0 \%$ & $0.0 \%$ & $0.5 \%$ & $0.0 \%$ & $13.9 \%$ & $5.3 \%$ & $9.4 \%$ & $13.2 \%$ \\
\hline$q_{T r p}$ & $0.2 \%$ & $0.0 \%$ & $6.2 \%$ & $0.0 \%$ & $0.0 \%$ & $0.0 \%$ & $0.5 \%$ & $0.0 \%$ & $20.0 \%$ & $2.2 \%$ \\
\hline$q_{T y r}$ & $21.8 \%$ & $0.0 \%$ & $41.7 \%$ & $2.6 \%$ & $0.0 \%$ & $0.0 \%$ & $5.5 \%$ & $0.1 \%$ & $28.3 \%$ & $5.3 \%$ \\
\hline$q_{V a l}$ & $12.5 \%$ & $5.1 \%$ & $34.1 \%$ & $0.0 \%$ & $0.6 \%$ & $6.8 \%$ & $3.6 \%$ & $0.0 \%$ & $17.1 \%$ & $14.2 \%$ \\
\hline
\end{tabular}


Across all other datasets, substantial increases in amino acid uptake rates are required to match the experimental values for $\mu_{g}$ and $q_{p}$. Except for both Martínez et al. (2015) Warm datasets, the uptake rate for amino acids which limit growth are auxotrophic (outlined in the bottom half of Table 2) and, in all but one case (Car HP NaBu), multiple auxotrophic amino acids limit the growth rate predictions. Particularly sharp deviations across multiple amino acids are observed for the Car LP and both Mart Warm datasets. In addition, both Mart Warm datasets are the only ones that require increased uptake of Gln.

Interestingly, when the upper bound for the Gln uptake flux is completely relaxed for the Mart Warm 2 dataset, a 74.5\% increase in uptake for this nutrient is obtained, and only Lys (required 14.2\% increase) and Thr (required 6.7\% increase) are observed to limit growth (data not shown). This result indicates that, for this dataset, the uptake rates of the remaining amino acids do not stoichiometrically limit biomass synthesis. Rather, the limitation in growth is associated with the relatively low uptake rate of Ser which, according to our model, can be overcome by Gluassociated biosynthesis via $F_{55}$. It is this additional intracellular Glu demand which pushes the uptake rates for Gln, Ile, Leu, Lys, and Val to higher values (Glu can be produced from these amino acids via reactions $F_{32}, F_{44}, F_{45}, F_{39}$, and $F_{41}$, respectively).

Full relaxation of the Gln uptake upper bound leads to a $65.9 \%$ increase and completely curbs the excess requirements for Ser. However, the excess demand for all remaining auxotrophic amino acids (His, Ile, Leu, Lys, Met, Phe, Trp, Tyr, and Val) is unchanged, indicating that for the Mart Cold 1 dataset, the uptake rates of auxotrophic amino acids stoichiometrically limit growth.

It is key to mention that underestimations for $\mu_{g}$ arise from two possible sources (or a combination thereof): (i) either the assumed stoichiometric coefficients for auxotrophic amino acids in biomass are too high or (ii) the measured uptake rates for auxotrophic amino acids is underestimated. Beyond typical experimental variability, the measured uptake rates are unlikely to have such drastic effects on the predicted growth (errors in uptake rate measurements above $30 \%$ are unlikely - see Table 2). Therefore, uncertainty associated with biomass composition is 

balance studies.

the more likely culprit, especially when considering that it has seldom been measured for flux

Questions surrounding CHO cell biomass composition have been recently addressed, where an average protein content $(\mathrm{w} / \mathrm{w})$ of $55.7 \% \pm 5.5 \%$ and a dry cell weight $(\mathrm{DCW})$ of $262.1 \pm 28.2$ $\mathrm{pg} / \mathrm{cell}$ is reported for multiple CHO cell lines cultured under different conditions (Szeliova et al., 2020). When comparing these values with the $350 \mathrm{pg} / \mathrm{cell}$ and $74.2 \% \mathrm{w} / \mathrm{w}$ protein assumed (not measured) for the datasets with the largest shortfalls in predicted $\mu_{g}$ (Martinez Warm), a maximum reduction of $\left(\frac{55.7 \%-5.5 \%}{74.2 \%}\right)\left(\frac{262.1 \frac{\mathrm{pg}}{\mathrm{cell}}-28.2 \frac{\mathrm{pg}}{\mathrm{cell}}}{350 \frac{\mathrm{pg}}{\mathrm{cell}}}\right)=45.3 \%$ in amino acid demand towards biomass synthesis can be computed. Such a reduction would compensate for the calculated shortfalls in auxotrophic amino acid uptake rates presented in Table 2 and, therefore, enhance the predictive capability of both our model and Hefzi's GeM.

Although detailed data for CHO cell biomass composition is now available (Szeliova et al., 2020), the values of $350 \mathrm{pg} /$ cell and $74.2 \% \mathrm{w} / \mathrm{w}$ protein were used for the results presented in Figure 2 and Table 2 in order to pinpoint how model predictive capability is impacted by our reduced reaction network when compared with the full GeM. In addition, Supplementary Figure 4 provides a comparison between the stoichiometric coefficients of amino acids in biomass assumed here with the experimentally determined ones from Szeliova et al. (2020). Despite considerable differences in DCW (219 pg/cell for exponential growth vs. $262.1 \pm 28.2 \mathrm{pg} / \mathrm{cell}$ ) and protein content $(74.2 \%$ vs. $55.7 \% \pm 5.5 \%)$, the stoichiometric coefficients result in quite comparable values. Therefore, we have elected to retain our originally assumed biomass composition for all subsequent calculations presented herein.

The above results highlight the importance of using accurate measurements for DCW and biomass composition in flux balance modelling. However, it is also important to mention that that biomass composition will impact model predictive capabilities only when auxotrophic components of biomass and product stoichiometrically limit growth and will be less of a factor 
when nutrient uptake rates exceed stoichiometric requirements. The latter case is observed for the Martinez Cold 2 dataset, where both the GeM and our model considerably over-predict $\mu_{g}$.

Several strategies have been developed to address situations where cell growth is not limited by stoichiometry. On one hand, exploring different metabolic objectives, such as maximising energetic efficiency, minimising redox stress or the uptake of essential nutrients (Chen et al., 2019; Feist and Palsson, 2010; Schuetz et al., 2007) can improve the predictive capability of flux models where nutrient uptake rates stoichiometrically exceed demand for growth and product synthesis. On another hand, strategies to further constrain the allowable values of fluxes through the reaction network have also been explored. For example, Lularevic et al. (2019) provide additional constraints using carbon balancing and Yeo et al. (2020) have developed an interesting framework whereby the fluxes through the reaction network are constrained based on the maximum rates and expression levels of the corresponding metabolic enzymes.

\subsection{CHOmpact facilitates easier interpretation of flux distributions.}

Our reduced reaction network and multi-objective optimisation strategy has two key advantages over full genome-scale models and the traditional growth rate maximisation objective function used to solve them. Firstly, our multi-objective optimisation strategy enables us to calculate flux distributions across different growth phases (beyond exponential growth) and, therefore, provides enhanced insight into flux distribution dynamics. In addition, the use of multiple objectives further constrains the solution space to achieve flux distributions that are biologically consistent. Secondly, our reduced reaction network simplifies model output interpretation and allows us to better relate the obtained flux distributions with cellular physiology.

\subsubsection{Flux distribution dynamics}

Figure 3 presents central carbon metabolism and Asp-Mal shuttle fluxes obtained with our reduced reaction network and multi-objective optimisation framework, where the flux maps correspond to three different feed compositions (Feed C, Feed U, and Feed U40) across the five phases of culture identified Supplementary Figure 2. 

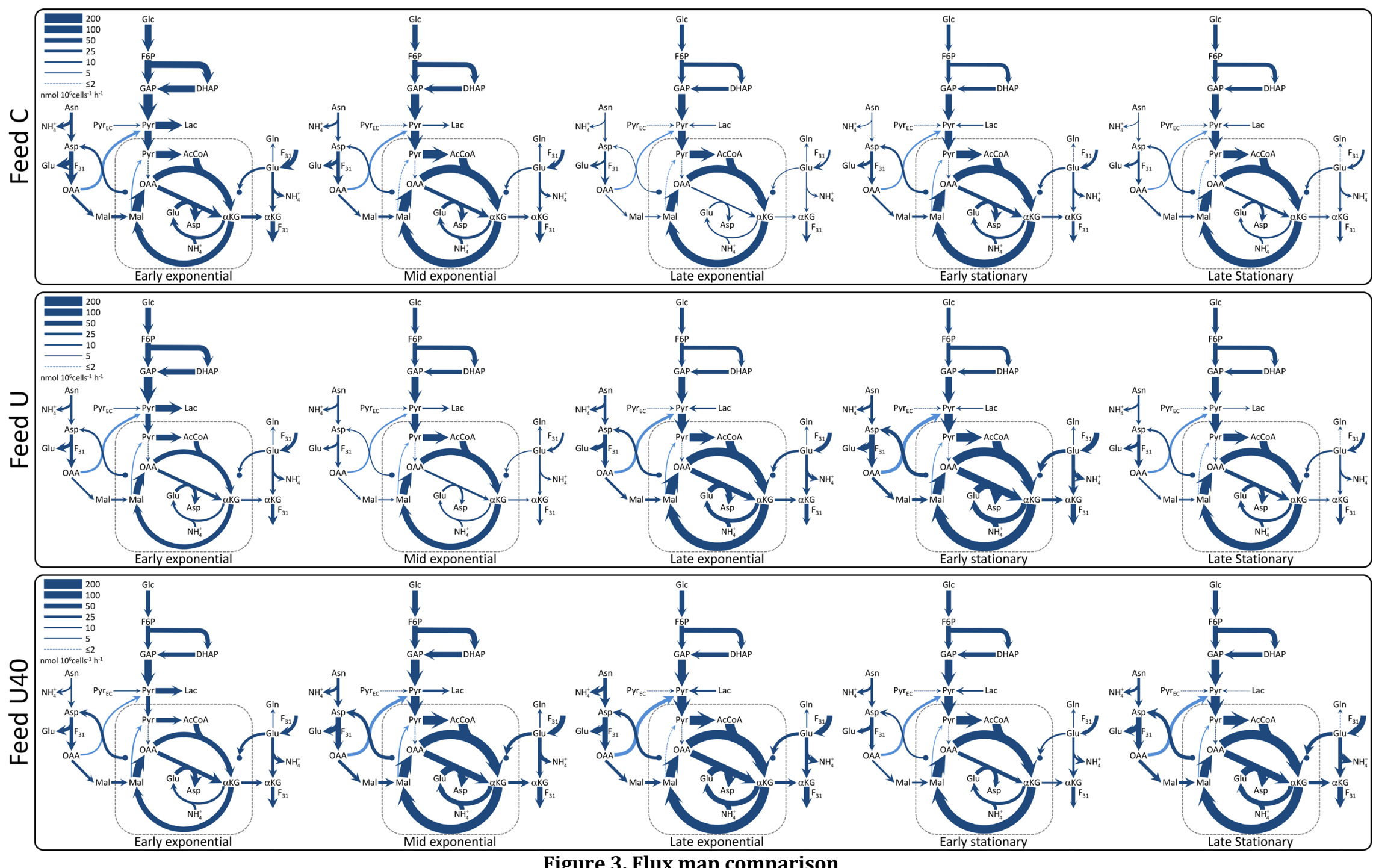

The flux distributions for central carbon metabolism and the aspartate/malate shuttle are shown for five culture intervals (early, mid, and late exponential as well as early and late stationary) across three feed compositions (Feed C, U, and U40). The line thickness corresponds to flux magnitude, as indicated by the legend at the top left-hand corner of each box. 
For all feed compositions, the glycolytic fluxes decrease with time, with the highest fluxes observed during early exponential growth and the lowest during stationary growth. The magnitude of the glycolytic flux is largely determined by the glucose uptake rates, which are shown to steadily decrease as culture progresses (Supplementary Figure 2). Conversely, the tricarboxylic acid (TCA) pathway fluxes increase with culture time, which occurs because reduced lactate production allows for more glycolysis-derived pyruvate to reach mitochondria. This phenomenon, often referred to as the Warburg effect, has been widely reported for rapidly proliferating cells, including CHO cells (Buchsteiner et al., 2018; Kelly et al., 2018).

The calculated fluxes through the Asp-Mal shuttle are defined by our imposed constraint on $F_{20}$

411 (Table 1), which limits its value to the flux of 'free' Glu (i.e., the sum of fluxes where this amino acid is produced which are not involved in the Asp-Mal shuttle). This constraint was set because all fluxes along the Asp-Mal shuttle cancel out and, therefore, can have any magnitude if the transfer of reducing equivalents from cytosolic to mitochondrial NADH is balanced. If left unconstrained, Asp-Mal shuttle fluxes have been reported to reach values that are comparable to those through central carbon metabolism (Mulukutla et al., 2012; Nolan and Lee, 2011). Although mathematically correct, these excessive flux values would be limited by the intracellular

418 availability of Glu, which is the key substrate for the rate-limiting Asp-Mal shuttle flux $\left(F_{20}\right)$

419 (LaNoue et al., 1974; LaNoue and Tischler, 1974). Despite not directly representing Glu availability, our proposed constraint does limit the upper values of Asp-Mal shuttle fluxes to ones that fall well below those of glycolysis and, therefore, make them more biologically consistent.

\subsubsection{Glutamate anaplerosis and cataplerosis}

423 Glutamate can either be consumed towards TCA and energy production (anaplerosis) or produced from TCA metabolites for subsequent use in biomass generation (cataplerosis).

425 According to our reaction network, net Glu anaplerosis occurs when more of this amino acid is 426 transported into mitochondria $\left(F_{20}\right)$ than what is transported out, in the form of $\alpha$ KG. Net Glu 
cataplerosis occurs when less of it is transported into mitochondria than the $\alpha \mathrm{KG}$ transported out. $\alpha \mathrm{KG}$ is then converted into Glu by either $F_{30}$ or $F_{31}$, the latter of which uses Asp as a co-substrate.

Figure 4 shows that, in Feed C, net Glu cataplerosis (Glu produced from TCA) is observed during the three exponential growth intervals. This trend is reversed during stationary phase, where net anaplerosis (Glu consumed towards TCA) occurs. Feed U and Feed U40 contrast with Feed C in 432 that they present Glu anaplerosis (consumption towards TCA) across all but one culture interval 433 (Figure 4 - bottom).

In Feed U, Glu cataplerosis is only observed during mid exponential growth, where its production from TCA accounts for $42.4 \%$ of the total Glu inlet (Figure 4 - top). Another interesting feature from Feed $U$ is the level of anaplerosis observed during the early stationary interval, where it accounts for $93.3 \%$ of the total consumed Glu (Figure 4 - bottom). This high level of anaplerosis is associated with increased Glu production from Gln (37.3\% of total) and, to a lesser extent, from His $(18.5 \%$ of total) (Figure $4-$ top). It is worth noting that this is the only culture condition and interval where Gln is consumed from the extracellular environment (Supplementary Figure 1). of all Glu produced is derived from TCA (Figure 4 - top). Glu cataplerosis during early stationary phase is most likely to arise to offset the high level of Gln secretion observed during this interval (Supplementary Figure 1).

In our GS-CHO Feed C cultures, Glu cataplerosis is driven by the high Asn uptake rates observed during exponential growth (Supplementary Figure 2), where a high $F_{31}$ flux provides a path for Asn overflow towards pyruvate via oaxaloacetate $\left(F_{29}\right)$. An alternative cataplerotic pathway for Glu is its direct synthesis from cytosolic $\alpha \mathrm{KG}$ via $F_{30}$ (catalysed by enzyme EC 1.4.1.3), which consumes $\mathrm{NH}_{4}{ }^{+}$and produces cytosolic $\mathrm{NAD}^{+}$(Yang et al., 2005) and may, thereby, reduce lactate production (Freund and Croughan, 2018). Its ability to reduce $\mathrm{NH}_{4}{ }^{+}$and lactate production make EC 1.4.1.3 a potential target for metabolic engineering. 


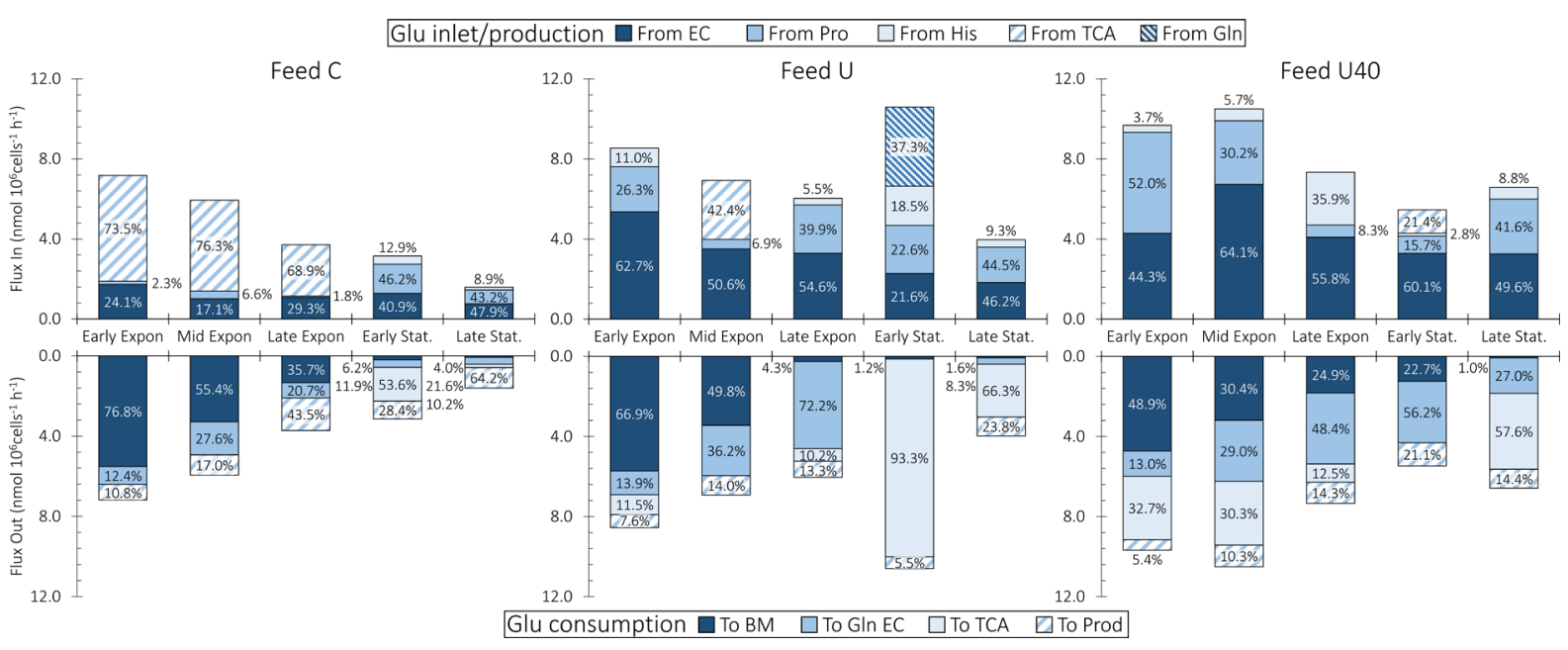

Figure 4. Glutamate flux distributions

The glutamate inlets for all feed compositions are shown in the top half and the outlets are shown in the bottom half. Glutamate sources and sinks are indicated by the shading and the top and bottom legends. The percent contributions to the total inlet or outlet are shown within the bars.

Prior flux balance work on standard (non-GS) CHO cells commonly reports net Glu anaplerosis during the exponential growth phase of cells, where high lactate production results in low TCA fluxes (Ahn and Antoniewicz, 2013). It is thought that Glu anaplerosis is used by the cells to replenish flux through TCA and is also known to be a major source of ammonia production because much of the anaplerotic flux involves glutamine aminolysis (Dean and Reddy, 2013; Wahrheit et al., 2014).

In contrast to past work, our FBA results indicate substantial Glu anaplerosis during the stationary phases of culture across all three feeding strategies (Figure 4 - bottom). Our results suggest that the high uptake rate of Asn observed across all cultures throttles Glu anaplerosis. The Asn overflow pathway discussed above produces Glu in $F_{31}$, which, in turn causes Glu overflow that is taken up by TCA. Anaplerosis as a means to cope with Glu overflow is also substantiated by the early stationary phase flux distribution of Feed $U$, which presents the highest level of Glu anaplerosis observed across all datasets (9.88 nmol/106 cells/h) (Figure 4 - bottom). This culture interval is the only one across all datasets where glutamine (Gln) is consumed by the cells. It is this Gln uptake which causes Glu overflow through the glutaminolysis pathway that is commonly reported for non-GS-CHO cells. 
Glu anaplerosis is commonly described as the cytosolic production of $\alpha \mathrm{KG}$ from Glu (via $F_{30}$ -

474 Figure 1), the former of which is transported into the mitochondrial matrix $\left(F_{21}\right)$ for uptake by 475 TCA (Ahn and Antoniewicz, 2013; Mulukutla et al., 2012; Nicolae et al., 2014). Crucially, the 476 transport of $\alpha \mathrm{KG}$ into the mitochondrial matrix depends on malate availability in the cytosol: $F_{21}$ 477 (OGCP) has an antiport mechanism whereby one molecule of $\alpha \mathrm{KG}$ is transported into the 478 mitochondrial lumen for every malate molecule that is transported out (Iacobazzi et al., 1992).

479 Our results indicate an alternative mechanism for Glu anaplerosis, where Glu is first transported 480 into the mitochondrial matrix (via $F_{20}$ - the rate-limiting and only irreversible reaction of the Asp481 Mal shuttle (LaNoue et al., 1974; LaNoue and Tischler, 1974)) where it is then converted, with the 482 consumption of mitochondrial Asp, into $\alpha$ KG via $F_{22}$. This alternative mechanism arises from the 483 high Asn uptake by our GS-CHO cells, where a substantial amount of this nutrient is funnelled 484 towards cytosolic malate through reactions $F_{51}, F_{31}$, and $F_{24}$. This Asn overflow pushes $\alpha \mathrm{KG}$ out 485 of the mitochondrial matrix where it can be consumed to produce Glu, mainly through $F_{31}$. Irrespective of the metabolic route, the magnitudes of Glu anaplerosis and cataplerosis are consistent with those determined through Metabolic Flux Analysis (Ahn and Antoniewicz, 2013; Nicolae et al., 2014).

\subsubsection{Asparagine and aspartate are key anaplerotic nutrients}

The asparagine/aspartate pair (Asn/Asp), linked through $F_{51}$, is a key contributor to TCA flux through its sequential conversion to oxaloacetate $\left(F_{31}\right)$ and pyruvate $\left(F_{29}\right)$, the latter of which is ultimately transported into mitochondria for consumption in the TCA. Because alanine and lactate are also produced from pyruvate (through reactions $F_{26}$ and $F_{25}$, respectively), the Asn/Asp pair is also a key source of these metabolites. towards TCA and 34.1\% towards lactate (Figure 5 - bottom). As lactate secretion subsides, the 

cultured with Feeds $\mathrm{U}$ and U40, where it exceeds $85 \%$ across the final three culture intervals.

500

501

502

503

504

505

506

507

508

509

510

511

512

513
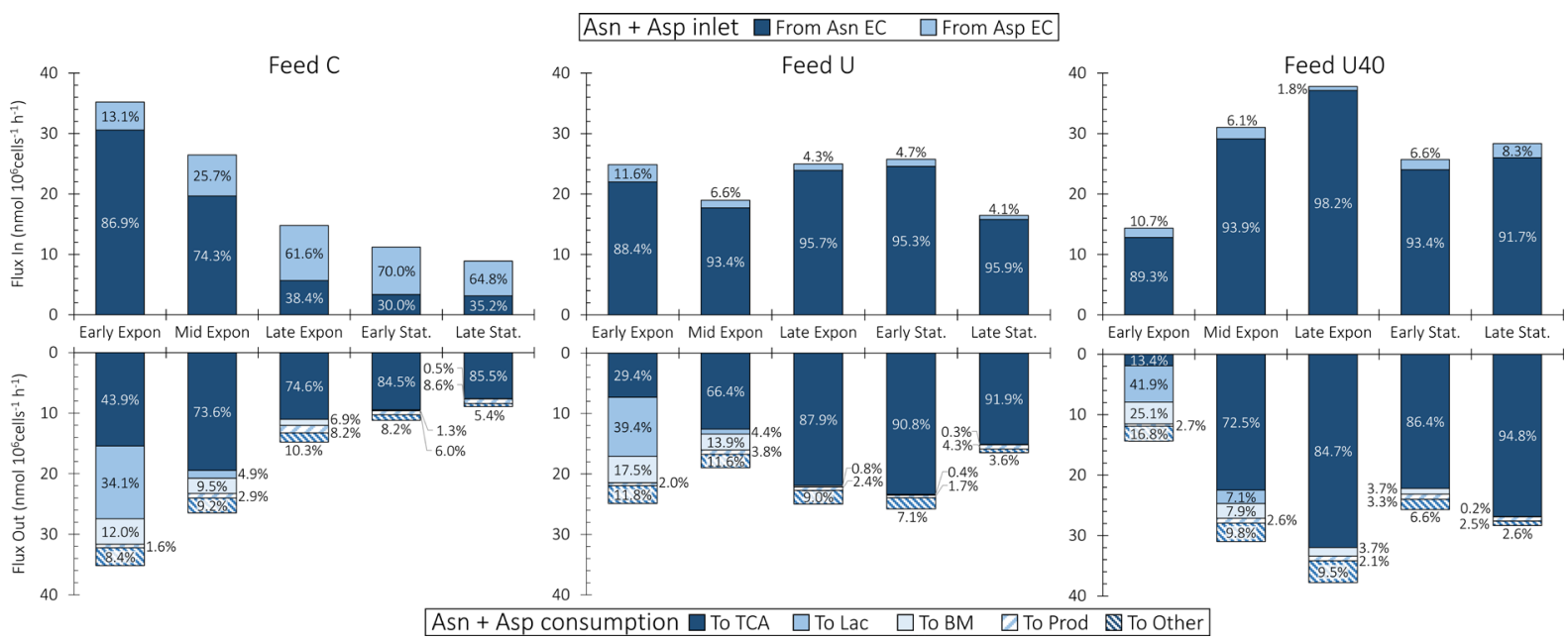

40 上

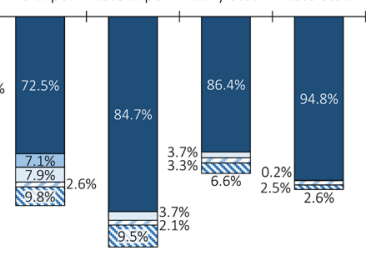

Figure 5. Asparagine + Aspartate flux distributions

The sources for asparagine (Asn) and aspartate (Asp) for all feed compositions is shown in the top half and the sinks are shown in the bottom half. The source and sink fluxes for Asn and Asp have been summed for simplicity. The different Asn+Asp sources and sinks are indicated by the shading and the top and bottom legends. The percent contributions to the total sources and sinks are shown within the bars.

Across all feeds, the maximum proportion of Asn/Asp consumed towards biomass and mAb product is $27.8 \%$ for Feed U40 during early exponential phase (Figure 5 - bottom). These results show that Asn/Asp are fed well beyond stoichiometric requirements for growth and product formation and that anaplerosis provides an overflow pathway for when these nutrients are fed in excess. These results are consistent with prior work where excess Asn/Asp feeding has been observed to increase alanine and lactate secretion by CHO cells (Calmels et al., 2019; Selvarasu et al., 2012).

The anaplerotic overflow pathway for Asn/Asp results in a rapid uptake of Asn and a concomitant reduction in its concentration in the culture medium (Supplementary Figure 1). Particularly low residual Asn concentrations are observed in the Feed C culture, where Asn and Asp are fed at the lowest levels. In absence of flux balance calculations, the observed reduction in Asn availability 
could be interpreted as being growth limiting and would lead to increasing the concentration of

519 Asn in the media and/or feed to alleviate the perceived bottleneck. Our experimental and FBA

520 results demonstrate that Asn is indeed not a growth limiting nutrient, and that increasing its

521 concentration in the feed in fact reduces cell growth (Supplementary Figure 1), likely due to the

522 production of ammonia associated with Asn anaplerosis. Similar observations were recently reported by Calmels et al. (2019), where GeM FBA calculations were applied to industrial CHO DG44 cells.

FBA also allows us to estimate the Asn/Asp uptake rates at which the anaplerotic pathway becomes saturated (i.e., where no more Asn/Asp can be funnelled towards TCA). The raw experimental data shows that Asp accumulates in the extracellular environment of Feed U40 cultures during the late exponential, early stationary, and stationary phases (Supplementary

Figure 1) implying that, under these conditions, the cells resort to secreting Asp instead of consuming it towards central carbon metabolism. The flux of Asn/Asp towards TCA during these culture phases are $38.7 \mathrm{nmol} / 10^{6} \mathrm{cells} / \mathrm{h}, 25.7 \mathrm{nmol} / 10^{6} \mathrm{cells} / \mathrm{h}$, and $32.1 \mathrm{nmol} / 10^{6} \mathrm{cells} / \mathrm{h}$, respectively and represent the range of Asn/Asp overflow our GS-CHO cells can cope with. Interestingly, these values closely correlate with the values of total available Glu flux, which are $7.3 \mathrm{nmol} / 10^{6} \mathrm{cells} / \mathrm{h}, 5.5 \mathrm{nmol} / 10^{6} \mathrm{cells} / \mathrm{h}$, and $6.6 \mathrm{nmol} / 10^{6} \mathrm{cells} / \mathrm{h}$ for the corresponding culture intervals. This correlation indicates that intracellular Glu availability may regulate the extent of Asn/Asp anaplerosis.

\subsection{4. $\mathrm{NH}_{4}{ }^{+}$sources and sinks}

$538 \mathrm{NH}_{4}+$ is a key determinant of $\mathrm{CHO}$ cell culture performance because it is known to impact cell growth (Synoground et al., 2021; Wahrheit et al., 2014) as well as product quality (Borys et al., 1994; Hong et al., 2010). In standard CHO cells, $\mathrm{NH}_{4}+$ is mainly generated as a by-product of Gln

541 anaplerosis (glutaminolysis) (Dean and Reddy, 2013; Hong et al., 2010; Wahrheit et al., 2014).

542 Glutamine synthase (GS) cells, such as the ones used in this study, satisfy their Gln requirements

543 by producing it from Glu via ectopic expression of glutamine synthase, thus circumventing the 
negative impact of $\mathrm{NH}_{4}{ }^{+}$on the cell culture process. Despite considerable reductions in $\mathrm{NH}_{4}{ }^{+}$ accumulation, GS-CHO cells still produce ammonia to levels that may still impact product glycosylation (Borys et al., 1994; Hong et al., 2010) so it is therefore important to characterise the major sources and sinks of this key metabolite.

The dark blue bars in the top half of Figure 6 show that the vast majority ( $>75 \%$ ) of ammonia is produced from asparagine $\left(F_{51}\right)$. The only exceptions are the Early Stationary intervals of Feed C and Feed $\mathrm{U}$ where, respectively, Asn is the source of $46.4 \%$ and $67.5 \%$ of all produced $\mathrm{NH}_{4}{ }^{+}$. During the Early Stationary phase of Feed C, the lower levels of $\mathrm{NH}_{4}+$ production from Asn are due to depletion of this nutrient in the culture media along with $\mathrm{NH}_{4}^{+}$uptake by the cells (Supplementary Figure 1). In Feed U, the lower proportion of $\mathrm{NH}_{4}{ }^{+}$generated from Asn is caused by glutaminolysis - this is the only interval across all experiments where Gln is consumed by the cells (Supplementary Figure 1$)$. Additional sources of $\mathrm{NH}_{4}+$ include Ser $\left(F_{36}\right)$, Thr $\left(F_{37}\right)$ and His $\left(F_{54}\right)$, although to much lower levels, when compared with Asn. These results indicate that, in GSCHO cells, $\mathrm{NH}_{4}^{+}$production is throttled by the Asn/Asp anaplerosis discussed in section 3.2 .3 and is consistent with previous work with GS-CHO cells (Calmels et al., 2019; Carinhas et al., 2013).

The total production rate of $\mathrm{NH}_{4}+$ further confirms its link with Asn/Asp anaplerosis. The top half of Figure 6 shows that the total amount of $\mathrm{NH}_{4}{ }^{+}$produced by the cells increases with higher levels of Asn feeding. After the Mid Exponential interval, cells cultured with basal Asn feeding levels (Feed C) have $\mathrm{NH}_{4}{ }^{+}$production rates below $8 \mathrm{nmol} / 10^{6} \mathrm{cells} / \mathrm{h}$, whereas the Feed $\mathrm{U}$ and Feed $\mathrm{U} 40$ cultures (increasingly higher levels of Asn feeding) produce above $20 \mathrm{nmol} / 10^{6} \mathrm{cells} / \mathrm{h}$ of $\mathrm{NH}_{4}{ }^{+}$.

The bottom half of Figure 6 presents the major $\mathrm{NH}_{4}{ }^{+}$sinks across the three feed conditions, as obtained through our FBA framework. There, it can be seen that three $\mathrm{NH}_{4}{ }^{+}$sinks predominate: (i) secretion to the extracellular (EC) environment (dark blue bars $-F_{108}$ ), (ii) consumption in mitochondria (intermediate blue bars) and (iii) consumption towards Gln synthesis (light blue bars $-\mathrm{F}_{32}$ ). Of the three major sinks, EC secretion can be deduced from the experimental data. The sink towards Gln synthesis is similarly intuitive and occurs because our GS-CHO cells are not fed 
570 Gln and must cover their demand for this amino acid through its GS-enabled synthesis using Glu 571 and $\mathrm{NH}_{4}{ }^{+}$as substrates.

572

573

574

575

576

577

578

579

580

581

582

583

584

585

586

587

588

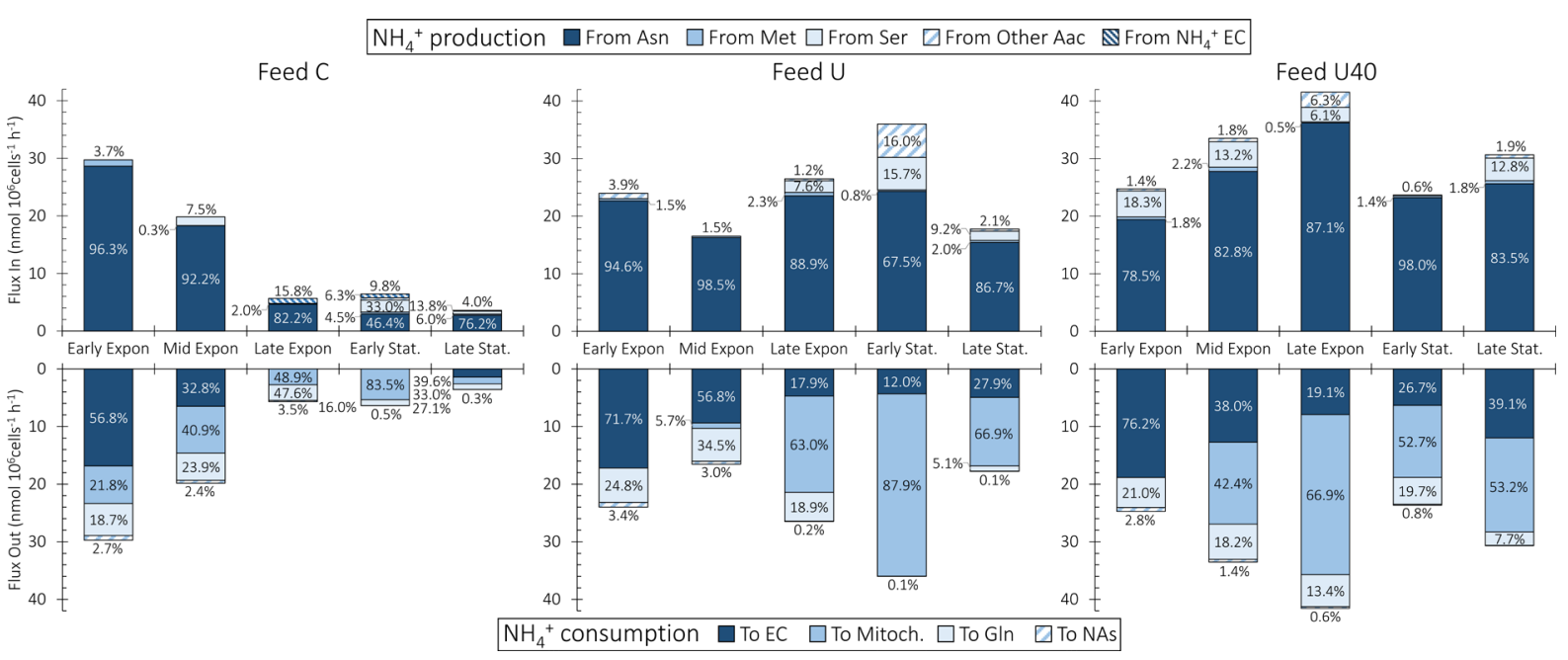

Figure 6. $\mathrm{NH}_{4}{ }^{+}$Flux distributions

The $\mathrm{NH}_{4}{ }^{+}$sources for all feed compositions are shown in the top half and the sinks are shown in the bottom half. The different sources and sinks are indicated by the shading and the top and bottom legends. The percent contribution of each source/sink to the total produced/consumed is shown within the bars.

The less intuitive $\mathrm{NH}_{4}{ }^{+}$sink is the one associated with mitochondria. According to our FBA results, a substantial amount of $\mathrm{NH}_{4}{ }^{+}$is consumed by a mitochondrial reaction cycle, where OAA is combined with Glu to produce $\alpha \mathrm{KG}$ and Asp $\left(F_{22}\right)$ and where the resulting $\alpha \mathrm{KG}$ is combined with $\mathrm{NH}_{4}+$ to produce Glu $\left(F_{23}\right)$. This mitochondrial $\mathrm{NH}_{4}{ }^{+}$sink is governed by the presence of Asp within the mitochondrial lumen and is therefore coupled with the Asp-Mal shuttle, where $F_{20}$ transports Asp out of mitochondria. If Asp accumulates within mitochondria, $F_{22}$ and $F_{23}$ will be reversed and may, thereby, cause net $\mathrm{NH}_{4}+$ production by mitochondria. These results are consistent with experimental findings where high $\mathrm{NH}_{4}{ }^{+}$concentrations increased the cell specific consumption rates of Asp and Glu (Lao and Toth, 1997).

Due to the constraint imposed on $F_{20}$ by our FBA solution strategy (discussed in Section 2.2.2 and presented in Table 1), a second sink for mitochondrial Asp is required to drive mitochondrial $\mathrm{NH}_{4}+$ consumption. Within our FBA reaction network, this additional sink is given by $F_{59}$ of the 
urea cycle, where mitochondrial Asp is irreversibly combined with citrulline to produce fumarate and arginine (Supplementary Table 1). Our results indicate that $F_{59}$ consumes over half of the mitochondrial Asp across all culture conditions (Supplementary Figure 5) and is, therefore, a key determinant of mitochondrial consumption of $\mathrm{NH}_{4}^{+}$.

594 The above mitochondrial Asp sink $\left(F_{59}\right)$ enables mitochondrial consumption of $\mathrm{NH}_{4}{ }^{+}$that is 595 independent of the Asp-Mal shuttle and, therefore, of cytosolic Glu availability. This Glu596 independent $\mathrm{NH}_{4}{ }^{+}$detoxification pathway would require diverting aKG directly from TCA to be 597 consumed in reaction $F_{23}$. The produced Glu would then react with TCA-derived oxaloacetate to 598 replenish aKG and produce mitochondrial Asp $\left(F_{22}\right)$. Finally, mitochondrial Asp would be consumed by $F_{59}$ to yield arginine and fumarate, which could also feed back into TCA.

Interestingly, $\mathrm{CHO}$ cells are reported to produce only trace amounts of urea (Zamorano et al., 2010), indicating that certain enzymes of the cycle may be inactive. The mitochondrial sink identified by our FBA may be an alternate route of $\mathrm{NH}_{4}{ }^{+}$detoxification (independent of urea secretion) that leverages two of the urea cycle enzymes (EC 6.3.4.5 and EC 4.3.2.1) which are known to be expressed in CHO cells (Heffner et al., 2020).

\section{Concluding remarks}

606 We have presented a reduced reaction network to describe the metabolism of mAb-producing 607 CHO cells. Our reduced metabolic network (144 reactions) performs comparably with the 608 iCHO1766 GeM (>6000 reactions) in predicting the growth rates of different CHO cell lines. Our 609 FBA framework also allowed us to identify the absence of cellular weight and composition measurements as the most likely cause of inaccuracies in predicting the growth rates.

611 We have also presented a comprehensive multi-objective optimisation strategy to solve our

612 metabolic model. Our multi-objective optimisation framework constrains the solution space to 613 yield physiologically consistent flux distributions across all phases of cell culture. 
614 When coupled with multi-objective optimisation, our compact reaction network greatly enhances

615 the interpretability of metabolic flux distributions across the different phases of cell culture. In

616 this context, our results provide insights into the mechanisms underlying Glu anaplerosis and its

617 dependence on the uptake rate of Asn/Asp. We have also identified Asn and Asp as the key

618 anaplerotic nutrients of GS-CHO cells and that, in this role, they are an important source of lactate

619 during the early stages of culture.

620 Our results also show that Asn is the predominant source of $\mathrm{NH}_{4}{ }^{+}$across all culture conditions

621 and that the major sink for this key metabolite is consumption within mitochondria. The presence

622 of Asp within mitochondria determines whether this organelle is a source or sink of $\mathrm{NH}_{4}^{+}$: when

623 Asp accumulates, mitochondria can become a net source of $\mathrm{NH}_{4}{ }^{+}$, when Asp is depleted, $\mathrm{NH}_{4}{ }^{+}$is

624 consumed within mitochondria. The Asp-Mal shuttle determines the intracellular flux 625 distributions of Asn, Asp, Gln, Glu and $\mathrm{NH}_{4}{ }^{+}$. Our FBA solution strategy constrains fluxes through 626 the Asp-Mal to not exceed the flux of 'free' Glu entering or produced by the cells in order to obtain physiologically consistent flux distributions for Asn, Asp, Gln, Glu and $\mathrm{NH}_{4}{ }^{+}$.

Moving forward, the enhanced understanding of metabolic dynamics afforded by our reduced reaction network and multi-objective optimisation framework can be used to define feeding strategies that optimise cell culture performance. Furthermore, the compact size of our reaction network will also facilitate the creation of hybrid dynamic FBA/culture dynamics models which can be used as digital twins for dynamic optimisation and control of cell culture bioprocesses.

\section{Acknowledgements}

634 CK and IJV gratefully acknowledge funding by BRIC/BBSRC. IJV also acknowledges funding from Science Foundation Ireland (12/RC/2275_P2). This manuscript is dedicated in memory of Aoife Dublin.

The authors declare no conflicts of interest. 
640

641

642

643

644

645

646

647

648

649

650

651

652

653

654

655

656

657

658

659

660

661

662

663

664

\section{References}

Ahn, W. S., Antoniewicz, M. R., 2013. Parallel labeling experiments with [1,2-(13)C]glucose and [U-(13)C]glutamine provide new insights into CHO cell metabolism. Metab Eng. 15, 34-47. https://doi.org/10.1016/i.ymben.2012.10.001.

Albrecht, S., Lane, J. A., Marino, K., Al Busadah, K. A., Carrington, S. D., Hickey, R. M., Rudd, P. M., 2014. A comparative study of free oligosaccharides in the milk of domestic animals. Br J Nutr. 111, 1313-28. https://doi.org/10.1017/S0007114513003772.

Banga, J. R., 2008. Optimization in computational systems biology. BMC Syst Biol. 2, 47. https://doi.org/10.1186/1752-0509-2-47.

Baycin-Hizal, D., Tabb, D. L., Chaerkady, R., Chen, L., Lewis, N. E., Nagarajan, H., Sarkaria, V., Kumar, A., Wolozny, D., Colao, J., Jacobson, E., Tian, Y., O'Meally, R. N., Krag, S. S., Cole, R. N., Palsson, B. O., Zhang, H., Betenbaugh, M., 2012. Proteomic analysis of Chinese hamster ovary cells. J Proteome Res. 11, 5265-76. https://doi.org/10.1021/pr300476w.

Borys, M. C., Linzer, D. I., Papoutsakis, E. T., 1994. Ammonia affects the glycosylation patterns of recombinant mouse placental lactogen-I by chinese hamster ovary cells in a pH-dependent manner. Biotechnol Bioeng. 43, 505-14. https://doi.org/10.1002/bit.260430611.

Brady, R. L., Hubbard, R. E., King, D. J., Low, D. C., Roberts, S. M., Todd, R. J., 1991. Crystallization and preliminary X-ray diffraction study of a chimaeric Fab' fragment of antibody binding tumour cells. J Mol Biol. 219, 603-4.

Buchsteiner, M., Quek, L. E., Gray, P., Nielsen, L. K., 2018. Improving culture performance and antibody production in $\mathrm{CHO}$ cell culture processes by reducing the Warburg effect. Biotechnol Bioeng. 115, 2315-2327. https://doi.org/10.1002/bit.26724.

Calmels, C., McCann, A., Malphettes, L., Andersen, M. R., 2019. Application of a curated genomescale metabolic model of CHO DG44 to an industrial fed-batch process. Metab Eng. 51, 9-19. https://doi.org/10.1016/i.ymben.2018.09.009. 
665 Campbell, M. P., Royle, L., Radcliffe, C. M., Dwek, R. A., Rudd, P. M., 2008. GlycoBase and autoGU: tools for HPLC-based glycan analysis. Bioinformatics. 24, 1214-6. https://doi.org/10.1093/bioinformatics/btn090.

668 Carinhas, N., Duarte, T. M., Barreiro, L. C., Carrondo, M. J., Alves, P. M., Teixeira, A. P., 2013. Metabolic signatures of GS-CHO cell clones associated with butyrate treatment and culture phase transition. Biotechnol Bioeng. 110, 3244-57. https://doi.org/10.1002/bit.24983.

Chen, Y., McConnell, B. O., Gayatri Dhara, V., Mukesh Naik, H., Li, C. T., Antoniewicz, M. R., Betenbaugh, M. J., 2019. An unconventional uptake rate objective function approach enhances applicability of genome-scale models for mammalian cells. NPJ Syst Biol Appl. 5, 25. https://doi.org/10.1038/s41540-019-0103-6.

Dean, J., Reddy, P., 2013. Metabolic analysis of antibody producing CHO cells in fed-batch production. Biotechnol Bioeng. 110, 1735-47. https://doi.org/10.1002/bit.24826.

del Val, I. J., Fan, Y., Weilguny, D., 2016a. Dynamics of immature mAb glycoform secretion during CHO cell culture: An integrated modelling framework. Biotechnol J. 11, 610-23. https://doi.org/10.1002/biot.201400663.

del Val, I. J., Polizzi, K. M., Kontoravdi, C., 2016b. A theoretical estimate for nucleotide sugar https://doi.org/10.1038/srep28547.

Fan, Y., del Val, I. J., Muller, C., Lund, A. M., Sen, J. W., Rasmussen, S. K., Kontoravdi, C., Baycin-Hizal, 685 D., Betenbaugh, M. J., Weilguny, D., Andersen, M. R., 2015. A multi-pronged investigation into the effect of glucose starvation and culture duration on fed-batch CHO cell culture. Biotechnol Bioeng. 112, 2172-84. https://doi.org/10.1002/bit.25620.

Feist, A. M., Palsson, B. O., 2010. The biomass objective function. Curr Opin Microbiol. 13, 344-9. https://doi.org/10.1016/j.mib.2010.03.003.

Fouladiha, H., Marashi, S. A., Li, S., Li, Z., Masson, H. O., Vaziri, B., Lewis, N. E., 2021. Systematically gap-filling the genome-scale metabolic model of CHO cells. Biotechnol Lett. 43, 73-87. 
Freund, N. W., Croughan, M. S., 2018. A Simple Method to Reduce both Lactic Acid and Ammonium Production in Industrial Animal Cell Culture. Int J Mol Sci. 19. https://doi.org/10.3390/ijms19020385.

Gardner, J. J., Boyle, N. R., 2017. The use of genome-scale metabolic network reconstruction to predict fluxes and equilibrium composition of $\mathrm{N}$-fixing versus $\mathrm{C}$-fixing cells in a diazotrophic cyanobacterium, Trichodesmium erythraeum. BMC $\quad$ Syst Biol. 11, 4. https://doi.org/10.1186/s12918-016-0383-z.

Gutierrez, J. M., Feizi, A., Li, S., Kallehauge, T. B., Hefzi, H., Grav, L. M., Ley, D., Baycin Hizal, D., Betenbaugh, M. J., Voldborg, B., Faustrup Kildegaard, H., Min Lee, G., Palsson, B. O., Nielsen, J., Lewis, N. E., 2020. Genome-scale reconstructions of the mammalian secretory pathway predict metabolic costs and limitations of protein secretion. Nat Commun. 11, 68. https://doi.org/10.1038/s41467-019-13867-y.

Heffner, K., Hizal, D. B., Majewska, N. I., Kumar, S., Dhara, V. G., Zhu, J., Bowen, M., Hatton, D., Yerganian, G., Yerganian, A., O'Meally, R., Cole, R., Betenbaugh, M., 2020. Expanded Chinese hamster organ and cell line proteomics profiling reveals tissue-specific functionalities. Sci Rep. 10, 15841. https://doi.org/10.1038/s41598-020-72959-8.

Hefzi, H., Ang, K. S., Hanscho, M., Bordbar, A., Ruckerbauer, D., Lakshmanan, M., Orellana, C. A., Baycin-Hizal, D., Huang, Y., Ley, D., Martinez, V. S., Kyriakopoulos, S., Jimenez, N. E., Zielinski, D. C., Quek, L. E., Wulff, T., Arnsdorf, J., Li, S., Lee, J. S., Paglia, G., Loira, N., Spahn, P. N., Pedersen, L. E., Gutierrez, J. M., King, Z. A., Lund, A. M., Nagarajan, H., Thomas, A., AbdelHaleem, A. M., Zanghellini, J., Kildegaard, H. F., Voldborg, B. G., Gerdtzen, Z. P., Betenbaugh, M. J., Palsson, B. O., Andersen, M. R., Nielsen, L. K., Borth, N., Lee, D. Y., Lewis, N. E., 2016. A Consensus Genome-scale Reconstruction of Chinese Hamster Ovary Cell Metabolism. Cell Syst. 3, 434-443 e8. https://doi.org/10.1016/j.cels.2016.10.020.

Heilig, R., Eckenberg, R., Petit, J. L., Fonknechten, N., Da Silva, C., Cattolico, L., Levy, M., Barbe, V., de Berardinis, V., Ureta-Vidal, A., Pelletier, E., Vico, V., Anthouard, V., Rowen, L., Madan, A., Qin, S., Sun, H., Du, H., Pepin, K., Artiguenave, F., Robert, C., Cruaud, C., Bruls, T., Jaillon, O., 

human chromosome 14. Nature. 421, 601-7. https://doi.org/10.1038/nature01348.

Hong, J. K., Cho, S. M., Yoon, S. K., 2010. Substitution of glutamine by glutamate enhances production and galactosylation of recombinant IgG in Chinese hamster ovary cells. Appl Microbiol Biotechnol. 88, 869-76. https://doi.org/10.1007/s00253-010-2790-1.

Iacobazzi, V., Palmieri, F., Runswick, M. J., Walker, J. E., 1992. Sequences of the human and bovine genes for the mitochondrial 2-oxoglutarate carrier. DNA Seq. 3, 79-88. https://doi.org/10.3109/10425179209034000.

Jeske, L., Placzek, S., Schomburg, I., Chang, A., Schomburg, D., 2019. BRENDA in 2019: a European ELIXIR core data resource. Nucleic Acids Res. 47, D542-D549. https://doi.org/10.1093/nar/gky1048.

Kanehisa, M., Furumichi, M., Tanabe, M., Sato, Y., Morishima, K., 2017. KEGG: new perspectives on genomes, pathways, diseases and drugs. Nucleic Acids Res. 45, D353-D361. https://doi.org/10.1093/nar/gkw1092.

Kanehisa, M., Sato, Y., Furumichi, M., Morishima, K., Tanabe, M., 2019. New approach for understanding genome variations in KEGG. Nucleic Acids Res. 47, D590-D595. 
Kelly, P. S., Alarcon Miguez, A., Alves, C., Barron, N., 2018. From media to mitochondria-rewiring cellular energy metabolism of Chinese hamster ovary cells for the enhanced production of biopharmaceuticals. Current Opinion in Chemical Engineering. 22, 71-80. https://doi.org/https://doi.org/10.1016/j.coche.2018.08.009.

Kol, S., Ley, D., Wulff, T., Decker, M., Arnsdorf, J., Schoffelen, S., Hansen, A. H., Jensen, T. L., Gutierrez, J. M., Chiang, A. W. T., Masson, H. O., Palsson, B. O., Voldborg, B. G., Pedersen, L. E., Kildegaard, H. F., Lee, G. M., Lewis, N. E., 2020. Multiplex secretome engineering enhances recombinant protein production and purity. Nat Commun. 11, 1908. https://doi.org/10.1038/s41467020-15866-w.

Kremkow, B. G., Baik, J. Y., MacDonald, M. L., Lee, K. H., 2015. CHOgenome.org 2.0: Genome resources and website updates. Biotechnol J. 10, 931-8. https://doi.org/10.1002/biot.201400646.

Kremkow, B. G., Lee, K. H., 2018. Glyco-Mapper: A Chinese hamster ovary (CHO) genome-specific $\begin{array}{lllll}\text { glycosylation } & \text { prediction } & \text { Metab } & \text { Eng. 47, }\end{array}$ https://doi.org/10.1016/j.ymben.2018.03.002.

Kyriakopoulos, S., Kontoravdi, C., 2014. A framework for the systematic design of fed-batch strategies in mammalian cell culture. Biotechnol Bioeng. 111, 2466-76. https://doi.org/10.1002/bit.25319.

LaNoue, K. F., Meijer, A. J., Brouwer, A., 1974. Evidence for electrogenic aspartate transport in rat liver mitochondria. Arch Biochem Biophys. 161, 544-50.

LaNoue, K. F., Tischler, M. E., 1974. Electrogenic characteristics of the mitochondrial glutamateaspartate antiporter. J Biol Chem. 249, 7522-8.

Lao, M. S., Toth, D., 1997. Effects of ammonium and lactate on growth and metabolism of a recombinant Chinese hamster ovary cell culture. Biotechnol Prog. 13, 688-91. https://doi.org/10.1021/bp9602360.

Lewis, N. E., Hixson, K. K., Conrad, T. M., Lerman, J. A., Charusanti, P., Polpitiya, A. D., Adkins, J. N., Schramm, G., Purvine, S. O., Lopez-Ferrer, D., Weitz, K. K., Eils, R., Konig, R., Smith, R. D., 
Palsson, B. O., 2010. Omic data from evolved E. coli are consistent with computed optimal growth from genome-scale models. Mol Syst Biol. 6, 390. https://doi.org/10.1038/msb.2010.47.

Lewis, N. E., Liu, X., Li, Y., Nagarajan, H., Yerganian, G., O'Brien, E., Bordbar, A., Roth, A. M., Rosenbloom, J., Bian, C., Xie, M., Chen, W., Li, N., Baycin-Hizal, D., Latif, H., Forster, J., Betenbaugh, M. J., Famili, I., Xu, X., Wang, J., Palsson, B. O., 2013. Genomic landscapes of Chinese hamster ovary cell lines as revealed by the Cricetulus griseus draft genome. Nat Biotechnol. 31, 759-65. https://doi.org/10.1038/nbt.2624.

Lularevic, M., Racher, A. J., Jaques, C., Kiparissides, A., 2019. Improving the accuracy of flux balance analysis through the implementation of carbon availability constraints for intracellular reactions. Biotechnol Bioeng. 116, 2339-2352. https://doi.org/10.1002/bit.27025.

Martínez, V. S., Buchsteiner, M., Gray, P., Nielsen, L. K., Quek, L.-E., 2015. Dynamic metabolic flux analysis using B-splines to study the effects of temperature shift on CHO cell metabolism. Metabolic Engineering Communications.

2 , 46-57. https://doi.org/https://doi.org/10.1016/j.meteno.2015.06.001.

Mulukutla, B. C., Gramer, M., Hu, W. S., 2012. On metabolic shift to lactate consumption in fedbatch culture of mammalian cells. Metab Eng. 14, 138-49. https://doi.org/10.1016/i.ymben.2011.12.006.

Nicolae, A., Wahrheit, J., Bahnemann, J., Zeng, A. P., Heinzle, E., 2014. Non-stationary 13C metabolic flux analysis of Chinese hamster ovary cells in batch culture using extracellular labeling highlights metabolic reversibility and compartmentation. BMC Syst Biol. 8, 50. https://doi.org/10.1186/1752-0509-8-50.

Nolan, R. P., Lee, K., 2011. Dynamic model of CHO cell metabolism. Metab Eng. 13, 108-24. https://doi.org/10.1016/i.ymben.2010.09.003.

O'Flaherty, R., Bergin, A., Flampouri, E., Mota, L. M., Obaidi, I., Quigley, A., Xie, Y., Butler, M., 2020. Mammalian cell culture for production of recombinant proteins: A review of the critical 
steps in their biomanufacturing. Biotechnol Adv. 43, 107552. https://doi.org/10.1016/j.biotechadv.2020.107552.

801

802

803

804

805

806

807

808

809

810

811

812

813

814

815

816

817

818

819

820

821

822

823

824

O'Leary, N. A., Wright, M. W., Brister, J. R., Ciufo, S., Haddad, D., McVeigh, R., Rajput, B., Robbertse, B., Smith-White, B., Ako-Adjei, D., Astashyn, A., Badretdin, A., Bao, Y., Blinkova, O., Brover, V., Chetvernin, V., Choi, J., Cox, E., Ermolaeva, O., Farrell, C. M., Goldfarb, T., Gupta, T., Haft, D., Hatcher, E., Hlavina, W., Joardar, V. S., Kodali, V. K., Li, W., Maglott, D., Masterson, P., McGarvey, K. M., Murphy, M. R., O'Neill, K., Pujar, S., Rangwala, S. H., Rausch, D., Riddick, L. D., Schoch, C., Shkeda, A., Storz, S. S., Sun, H., Thibaud-Nissen, F., Tolstoy, I., Tully, R. E., Vatsan, A. R., Wallin, C., Webb, D., Wu, W., Landrum, M. J., Kimchi, A., Tatusova, T., DiCuccio, M., Kitts, P., Murphy, T. D., Pruitt, K. D., 2016. Reference sequence (RefSeq) database at NCBI: current status, taxonomic expansion, and functional annotation. Nucleic Acids Res. 44, D733-45. https://doi.org/10.1093/nar/gkv1189.

Orth, J. D., Thiele, I., Palsson, B. O., 2010. What is flux balance analysis? Nat Biotechnol. 28, 245-8. https://doi.org/10.1038/nbt.1614.

Process Systems Enterprise, gPROMS. www.psenterprise.com/gproms. 1997-2020.

Rupp, O., MacDonald, M. L., Li, S., Dhiman, H., Polson, S., Griep, S., Heffner, K., Hernandez, I., Brinkrolf, K., Jadhav, V., Samoudi, M., Hao, H., Kingham, B., Goesmann, A., Betenbaugh, M. J., Lewis, N. E., Borth, N., Lee, K. H., 2018. A reference genome of the Chinese hamster based on a hybrid assembly strategy. Biotechnol Bioeng. 115, 2087-2100. https://doi.org/10.1002/bit.26722.

Sauer, P. W., Burky, J. E., Wesson, M. C., Sternard, H. D., Qu, L., 2000. A high-yielding, generic fedbatch cell culture process for production of recombinant antibodies. Biotechnol Bioeng. 67, 585-97.

Schuetz, R., Kuepfer, L., Sauer, U., 2007. Systematic evaluation of objective functions for predicting intracellular fluxes in Escherichia coli. Mol Syst Biol. 3, 119. https://doi.org/10.1038/msb4100162. 
825

826

827

828

829

830

831

832

833

834

835

836

837

838

840

841

842

843

844

845

846

847

848

849

850

851

Selvarasu, S., Ho, Y. S., Chong, W. P., Wong, N. S., Yusufi, F. N., Lee, Y. Y., Yap, M. G., Lee, D. Y., 2012. Combined in silico modeling and metabolomics analysis to characterize fed-batch CHO cell culture. Biotechnol Bioeng. 109, 1415-29. https://doi.org/10.1002/bit.24445.

Sheikh, K., Forster, J., Nielsen, L. K., 2005. Modeling hybridoma cell metabolism using a generic genome-scale metabolic model of Mus musculus. Biotechnol Prog. 21, 112-21. https://doi.org/10.1021/bp0498138.

Stockmann, H., Adamczyk, B., Hayes, J., Rudd, P. M., 2013. Automated, high-throughput IgGantibody glycoprofiling platform. Anal Chem. 85, 8841-9. https://doi.org/10.1021/ac402068r.

Synoground, B. F., McGraw, C. E., Elliott, K. S., Leuze, C., Roth, J. R., Harcum, S. W., Sandoval, N. R., 2021. Transient ammonia stress on Chinese hamster ovary (CHO) cells yield alterations to alanine metabolism and IgG glycosylation profiles. Biotechnol J. 16, e2100098. https://doi.org/10.1002/biot.202100098.

Szeliova, D., Ruckerbauer, D. E., Galleguillos, S. N., Petersen, L. B., Natter, K., Hanscho, M., Troyer, C., Causon, T., Schoeny, H., Christensen, H. B., Lee, D. Y., Lewis, N. E., Koellensperger, G., Hann, S., Nielsen, L. K., Borth, N., Zanghellini, J., 2020. What CHO is made of: Variations in the biomass composition of Chinese hamster ovary cell lines. Metab Eng. https://doi.org/10.1016/i.ymben.2020.06.002.

Wahrheit, J., Nicolae, A., Heinzle, E., 2014. Dynamics of growth and metabolism controlled by glutamine availability in Chinese hamster ovary cells. Appl Microbiol Biotechnol. 98, 177183. https://doi.org/10.1007/s00253-013-5452-2.

Xiang, J., Prasad, L., Delbaere, L. T., Jia, Z., 1999. Light-chain framework region residue Tyr71 of chimeric B72.3 antibody plays an important role in influencing the TAG72 antigen binding. Protein Eng. 12, 417-21.

Yang, S. J., Cho, E. H., Choi, M. M., Lee, H. J., Huh, J. W., Choi, S. Y., Cho, S. W., 2005. Critical role of the cysteine 323 residue in the catalytic activity of human glutamate dehydrogenase isozymes. Mol Cells. 19, 97-103. 
852 Yeo, H. C., Hong, J., Lakshmanan, M., Lee, D. Y., 2020. Enzyme capacity-based genome scale modelling of $\quad \mathrm{CHO}$ cells. Metab Eng.

60 , 138-147. https://doi.org/10.1016/j.ymben.2020.04.005.

855 Zamorano, F., Wouwer, A. V., Bastin, G., 2010. A detailed metabolic flux analysis of an underdetermined network of CHO cells. J Biotechnol. 150, 497-508. https://doi.org/10.1016/j.jbiotec.2010.09.944. 